\title{
MINIMAL STABILIZATION FOR DISCONTINUOUS GALERKIN FINITE ELEMENT METHODS FOR HYPERBOLIC PROBLEMS
}

\author{
E. BURMAN AND B. STAMM
}

\begin{abstract}
We consider a discontinuous Galerkin finite element method for the advection-reaction equation in two space-dimensions. For polynomial approximation spaces of degree greater than or equal to two on triangles we propose a method where stability is obtained by a penalization of only the upper portion of the polynomial spectrum of the jump of the solution over element edges. We prove stability in the standard $h$-weighted graphnorm and obtain optimal order error estimates with respect to mesh-size.
\end{abstract}

Discontinuous Galerkin method, advection-reaction equation, local mass conservation, interior penalty.

\section{INTRODUCTION}

The discontinuous Galerkin method (DG) for hyperbolic equations was introduced by Reed and Hill [21]. The method was then analysed in the framework of Friedrichs systems by Lesaint and Raviart [20]. A sharpened analysis was provided by Johnson and Pitkäranta [19]. During the nineties the discontinuous Galerkin method experienced a further development in the work by Cockburn and Shu where numerical schemes for hyperbolic problems were proposed by combining discontinuous Galerkin type approximation in space with Runge-Kutta type time stepping strategies $[9,10,11]$. A DG-method using high order approximation spaces was analysed by Houston, Schwab and Süli in [17]. In particular they proved quasioptimal $h p$-error estimates for hyperbolic problems. More recently the case of Friedrichs systems was revisited in the thesis of Jensen [18] and by Ern and Guermond [14]. In all the above works the basic strategy is the same: consider a polynomial approximation on each element and impose continuity weakly by adding a penalization term on the jump of the solution over interelement boundaries or equivalently choosing a numerical flux that has a dissipative property. The penalization can take the form of a so called upwind flux which corresponds to weak imposition of continuity on the inflow boundary or, as was pointed out by Brezzi and coworkers in [3], can be written as one term on the faces that assures positivity of the convective term and another term which is a pure penalization of the solution jumps. For a certain choice of the stabilization parameter in the latter case the two stabilizations coincide. An overview of different stabilization mechanisms in DG methods was recently proposed by Brezzi and coworkers in [1].

In parallell to the development of DG-methods for hyperbolic equations a method using continuous approximation but stabilizing the jump of the gradient over element edges has been proposed by Burman and Hansbo in [6] drawing on earlier ideas

The second author was supported by the Swiss National Science Foundation under grant $200021-113304$. 
of Douglas and Dupont [12]. This interior penalty method using continuous approximation spaces was then generalized by Burman to the case of non-conforming approximation spaces in [4] and to the $h p$-framework by Burman and Ern in [5].

In the recent paper [7] we studied theoretically and numerically what type of interior penalty stabilization is needed to obtain a stable, optimally converging scheme in the case of continuous or discontinuous approximation. Rather surprisingly we found that for a DG method using piecewise quadratic polynomials it was sufficient to stabilize the jump in the tangential derivative of the solution only. An optimal order a priori error estimate was proved and it was shown that for this method the local mass conservation is independent of the stabilization parameter of the numerical scheme, which is in general not the case for DG-methods.

In this paper we extend these ideas to the case of general polynomial order. In particular we prove that on triangular conforming meshes only the highest polynomial orders of the solution jumps need to be penalized. This can be considered as applying a high pass filter to the solution jumps before penalization or more precisly, projecting the jump onto the subspace consisting of the highest modes. Hence low order modes (approximately the lowest third of the polynomial spectrum) are not directly affected by the penalization term. This property leads to local mass conservation independent of the stabilization parameter. This is in general not true for DG-methods and when it does hold it often comes with decreased accuracy. Here we show both theoretically and numerically that our method leads to local mass conservation independently of the stabilization parameter without loss of accuracy. Shifting the numerical dissipation to higher order polynomial modes can be seen as a realization in the DG-framework of a spectral viscosity type of stabilization: low order modes will propagate without any explicit dissipation. For a discussion of minimal stabilization procedures in the framework of continuous approximation spaces see [2] and [4].

The main idea behind the proof is to construct a projection operator with orthogonality properties both on the elements and on the element faces. In this paper we restrict the analysis to the case of a linear scalar hyperbolic problem in two space dimensions to keep down redundant technical detail, however the same analysis is expected to carry over in a straightforward manner to more general first order systems such as symmetric Friedrichs systems in the framework proposed in [14], the wave equation or Maxwell's equations.

An outline of the paper is as follows: In the next section we first introduce the model problem and define our notation, then we give a serie of technical results concerning the projection operator used in the stability analysis. In section 3 we propose a discontinuous Galerkin method based on stabilization of the projected jumps and give some elementary lemmas. The proposed method is then analysed in section 4, where the main result is a discrete inf-sup condition showing that we may recover a priori control of the whole solution jump in the $L^{2}$-norm despite the fact that we only stabilize the highest modes of the jump. Once we have established the discrete inf-sup condition an $h$-optimal convergence analysis follows in a standard fashion. In section 5 , we give a full analysis of the projection introduced in section 2 . Finally, in section 6 , we show the numerical performance of our method on some simple model problems with varying regularity. 


\section{The PRoblem Setting}

Let $\Omega$ be an polygon in $\mathbb{R}^{2}$ with outer normal $n$. We consider the following advection-reaction equation with homogeneous Dirichlet boundary conditions on the inflow boundary:

Find $u: \Omega \rightarrow \mathbb{R}$ such that

$$
\left\{\begin{aligned}
\beta \cdot \nabla u+\mu u & =f, \\
\left.u\right|_{\partial \Omega^{-}} & =0,
\end{aligned}\right.
$$

where $f \in L^{2}(\Omega)$ and the vector field $\beta \in[\operatorname{Lip}(\Omega)]^{2}$ is supposed to be Lipschitz continuous. Assume that $\mu-\frac{1}{2} \nabla \cdot \beta \geq \mu_{0}>0$. The inflow boundary is defined by $\partial \Omega^{-}=\{x \in \partial \Omega ; \beta(x) \cdot n(x)<0\}$. For a discussion about the well-posedness of this problem we refer to [14].

2.1. Definitions. Let $\mathcal{K}$ be a subdivision of $\Omega \subset \mathbb{R}^{2}$ into non-overlapping triangles. Assume that $\mathcal{K}$ is shape-regular. For an element $\kappa \in \mathcal{K}, h_{\kappa}$ denotes its diameter. Set $h=\max _{\kappa \in \mathcal{K}} h_{\kappa}$ and let $\tilde{h}$ be the function such that $\left.\tilde{h}\right|_{\kappa}=h_{\kappa}$. Assume that $\mathcal{K}$ covers $\bar{\Omega}$ exactly and that $\mathcal{K}$ does not contain any hanging nodes. Suppose that each $\kappa \in \mathcal{K}$ is an affine image of the reference element $\widehat{\kappa}$. Let $\mathcal{F}_{i}$ denote the set of interior faces (1-manifolds) of the mesh, i.e., the set of faces that are not included in the boundary $\partial \Omega$. The set $\mathcal{F}_{e}$ denotes the faces that are included in $\partial \Omega$ and define $\mathcal{F}=\mathcal{F}_{i} \cup \mathcal{F}_{e}$. In addition we split the exterior boundary in an inflow and an outflow boundary, i.e. $\mathcal{F}_{e}=\mathcal{F}_{-} \cup \mathcal{F}_{+}$, where $\mathcal{F}_{ \pm}=\left\{F \in \mathcal{F}_{e} ; \pm \beta(x) \cdot n(x)>0 \forall x \in F\right\}$. For a face $F \in \mathcal{F}, h_{F}$ denotes its length and let $\tilde{h}_{\mathcal{F}}$ be the function such that $\left.\tilde{h}_{\mathcal{F}}\right|_{F}=h_{F}$.

For $s \geq 0$, let $H^{s}(\mathcal{K})$ be the space of piecewise Sobolev $H^{s}$-functions and denote its scalar product and norm by $(\cdot, \cdot)_{s, \mathcal{K}}$ resp. $\|\cdot\|_{s, \mathcal{K}}$. In the case of $s=0$ the subscript $s$ is dropped. For a subset $R \subset \mathcal{F}$ or $R \subset \mathcal{K},(\cdot, \cdot)_{R}$ denotes the $L^{2}(R)_{-}$ scalar product and $\|\cdot\|_{R}=(\cdot, \cdot)_{R}^{1 / 2}$ the corresponding norm.

For $v \in H^{1}(\mathcal{K})$ and an interior face $F=\kappa_{1} \cap \kappa_{2} \in \mathcal{F}_{i}$, where $\kappa_{1}$ and $\kappa_{2}$ are two distinct elements of $\mathcal{K}$ with respective outer normals $n_{1}$ and $n_{2}$, define the jump by

$$
[v]_{\beta}=\left(\left.v\right|_{\kappa_{1}} n_{1}+\left.v\right|_{\kappa_{2}} n_{2}\right) \cdot e_{\beta},
$$

where $e_{\beta}=\frac{\beta}{|\beta|}$ with $|\beta|=\sqrt{\beta \cdot \beta}$. The average is defined for all functions $v \in H^{1}(\mathcal{K})$ by

$$
\{v\}=\frac{1}{2}\left(\left.v\right|_{\kappa_{1}}+\left.v\right|_{\kappa_{2}}\right) .
$$

On outer faces $F=\partial \kappa \cap \partial \Omega \in \mathcal{F}_{e}$ with outer normal $n$, the jump and the average are defined as $[v]_{\beta}=\left.v\right|_{\kappa} n \cdot e_{\beta}$ and $\{v\}=\left.v\right|_{\kappa}$.

The shape-regularity implies that there exists a constant $c>0$ independent of the mesh size $h$ such that on any face $F \in \mathcal{F}$

$$
h_{F} \leq\{\tilde{h}\} \leq c h_{F} .
$$

In this paper $c>0$ denotes a generic constant and can change at each occurrence, while an indexed constant stays fix. Any constant is independent of the mesh size $h$. 
2.2. Projections and finite element spaces. Let us first define some polynomial spaces. Let $p \geq 0$ and $l \geq 0$ be two arbitrary integers and let $\kappa$ be an arbitrary element of the mesh $\mathcal{K}$. Further let $\mathbb{P}_{p}(\kappa)$ be the space of polynomials of total degree $p$ on $\kappa$ and introduce the global discontinuous finite element space

$$
V_{h}^{p}=\left\{v_{h} \in L^{2}(\Omega) ;\left.v_{h}\right|_{\kappa} \in \mathbb{P}_{p}(\kappa), \forall \kappa \in \mathcal{K}\right\}
$$

Define the following polynomial space on $\partial \kappa$ :

$$
\mathbb{P}_{l}(\partial \kappa)=\left\{v \in L^{2}(\partial \kappa) ;\left.v\right|_{F} \in \mathbb{P}_{l}(F), \forall F \in \mathcal{F}(\partial \kappa)\right\}
$$

where $\mathbb{P}_{l}(F)$ is the usual one dimensional polynomial space of total degree $l$ on $F$. $\mathcal{F}(\partial \kappa)$ denotes the set of faces of $\kappa$. Observe that no continuity is required on the vertices of $\kappa$. On a global level we define

$$
W_{h}^{l}=\left\{v \in L^{2}(\mathcal{F}) ;\left.v\right|_{F} \in \mathbb{P}_{l}(F), \forall F \in \mathcal{F}\right\}
$$

Associated to $W_{h}^{l}$, define the $L^{2}$-projection $P_{l}: L^{2}(\mathcal{F}) \rightarrow W_{h}^{l}$ by

$$
\left(P_{l} v, z_{h}\right)_{\mathcal{F}}=\left(v, z_{h}\right)_{\mathcal{F}} \quad \forall z_{h} \in W_{h}^{l}
$$

Consequently we have the following property for any function $z_{h} \in W_{h}^{l}$ :

$$
\left(\left(I-P_{l}\right) v, z_{h}\right)_{F}=0 \quad \forall F \in \mathcal{F} .
$$

Since $P_{l}$ is the facewise $L^{2}$-projection of order $l$ we have the following estimates

$$
\left\|w_{h} P_{l} v\right\|_{\mathcal{F}}^{2} \leq\left\|w_{h} v\right\|_{\mathcal{F}}^{2} \quad \text { and } \quad\left\|w_{h}\left(I-P_{l}\right) v\right\|_{\mathcal{F}}^{2} \leq\left\|w_{h} v\right\|_{\mathcal{F}}^{2} \quad \forall w_{h} \in W_{h}^{0} .
$$

Proposition 2.1 (Global Projection). Let $v_{1} \in L^{2}(\Omega)$ and $v_{2} \in L^{2}(\mathcal{F})$, then there exists a projection $\Pi_{h}=\Pi_{h}\left(v_{1}, v_{2}\right) \in V_{h}^{p}$, with $p \geq 2$, such that

$$
\begin{aligned}
\int_{\mathcal{K}}\left(\Pi_{h}-v_{1}\right) w_{h}=0 & \forall w_{h} \in V_{h}^{p-1}, \\
\int_{\mathcal{F}}\left(\left\{\Pi_{h}\right\}-v_{2}\right) z_{h}=0 & \forall z_{h} \in W_{h}^{l},
\end{aligned}
$$

for all $0 \leq l \leq\left\lfloor\frac{p+1}{3}\right\rfloor-1$. In addition for all $v \in L^{2}(\mathcal{F})$ the projection satisfies the following local stability property

$$
\left\|\Pi_{h}(0, v)\right\|_{\partial \kappa}^{2} \leq c\|v\|_{\partial \kappa}^{2},
$$

and its global variants

$$
\begin{aligned}
\left\|\left\{\Pi_{h}(0, v)\right\}\right\|_{\mathcal{F}}^{2}+\left\|\left[\Pi_{h}(0, v)\right]_{\beta}\right\|_{\mathcal{F}}^{2} & \leq c\|v\|_{\mathcal{F}}^{2}, \\
\left\|\Pi_{h}(0, v)\right\|_{\mathcal{K}}^{2} & \leq c\left\|\tilde{h}_{\mathcal{F}}^{\frac{1}{2}} v\right\|_{\mathcal{F}}^{2} .
\end{aligned}
$$

Proof. The proof of Proposition 2.1 is given in section 5 .

Remark 2.1. The result of (2.9) can be generalized by

$$
\left\|w_{h} \Pi_{h}(0, v)\right\|_{\mathcal{K}}^{2} \leq c\left\|\tilde{h}_{\mathcal{F}}^{\frac{1}{2}}\left\{w_{h}\right\} v\right\|_{\mathcal{F}}^{2}, \quad \forall w_{h} \in V_{h}^{0} .
$$


2.3. Some technical lemmas. In this section we present some known lemmas. The first is a generalized trace inequality and the second a standard inverse inequality. The proofs of these results can be found in textbooks such as [22] and [8].

Lemma 2.2 (Trace inequality). Let $\zeta_{h} \in\left[V_{h}^{p}\right]^{m}, m \geq 1$, then there exists a constant $c_{T}>0$, independent of the mesh size $h$, such that

$$
\left\|\left\{\zeta_{h}\right\}\right\|_{\mathcal{F}}^{2}+\left\|\left[\zeta_{h}\right]_{\beta}\right\|_{\mathcal{F}}^{2} \leq c_{T}\left\|\tilde{h}^{-\frac{1}{2}} \zeta_{h}\right\|_{\mathcal{K}}^{2} .
$$

On the other hand if $\zeta \in H^{1}(\mathcal{K})$, then there exists a constant $c_{T}>0$, independent of the mesh size $h$, such that

$$
\|\{\zeta\}\|_{\mathcal{F}}^{2}+\left\|[\zeta]_{\beta}\right\|_{\mathcal{F}}^{2} \leq c_{T}\left(\left\|\tilde{h}^{-\frac{1}{2}} \zeta\right\|_{\mathcal{K}}^{2}+\left|\tilde{h}^{\frac{1}{2}} \zeta\right|_{1, \mathcal{K}}^{2}\right) .
$$

Lemma 2.3 (Inverse inequality). Let $v_{h} \in V_{h}^{p}$, then there exists a constant $c>0$, independent of the mesh size $h$, such that

$$
\left\|\nabla v_{h}\right\|_{\mathcal{K}}^{2} \leq c\left\|\tilde{h}^{-1} v_{h}\right\|_{\mathcal{K}}^{2} .
$$

\section{The discontinuous finite Element method}

In this and further sections we restrict the choice of the polynomial order of the approximation to $p \geq 2$. The discrete problem consists of seeking $u_{h} \in V_{h}^{p}$ such that

$$
a\left(u_{h}, v_{h}\right)+j\left(u_{h}, v_{h}\right)=\left(f, v_{h}\right)_{\mathcal{K}} \quad \forall v_{h} \in V_{h}^{p}
$$

where

$$
\begin{aligned}
a(v, w) & =((\mu-\nabla \cdot \beta) v, w)_{\mathcal{K}}-(v, \beta \cdot \nabla w)_{\mathcal{K}}+\left(|\beta|\{v\},[w]_{\beta}\right)_{\mathcal{F}_{i} \cup \mathcal{F}_{+}}, \\
j(v, w) & =\gamma_{s}\left(|\beta|_{\infty}\left(I-P_{l}\right)[v]_{\beta},\left(I-P_{l}\right)[w]_{\beta}\right)_{\mathcal{F}_{i} \cup \mathcal{F}_{-}},
\end{aligned}
$$

and $l=\left\lfloor\frac{p+1}{3}\right\rfloor-1$. $P_{l}$ denotes the projection defined in section $2.2, \gamma_{s}$ denotes a stabilization parameter and $|\beta|_{\infty} \in W_{h}^{0}$ is defined by $\left.|\beta|_{\infty}\right|_{F}=\|\beta\|_{L^{\infty}(F)}$ on all faces $F \in \mathcal{F}$.

Remark 3.1 (Local mass conservation). Considering the model problem (2.1) with $\nabla \cdot \beta \equiv 0$ leads to the following local mass conservation property choosing the characteristic function of an element $\kappa \in \mathcal{K}$ as test function:

$$
\int_{\kappa} \mu u_{h}+\int_{\partial \kappa} \beta \cdot n_{\kappa}\left\{u_{h}\right\}=\int_{\kappa} f .
$$

Here $n_{\kappa}$ denotes the outer normal of the element $\kappa$. Observe that this property does not depend on the stabilization parameter $\gamma_{s}$ and can be considered as generalization of the exact conservation property for double-valued functions.

Remark 3.2 (Efficient implementation). Using the Bramble-Hilbert lemma one easily shows that the $\left(I-P_{l}\right)$ operator may be replaced by a differential operator of order $l+1$ in the tangential directions of the face. In particular when $l=0$ we get

$$
\left\||\beta|_{\infty}^{\frac{1}{2}}\left(I-P_{0}\right)[v]_{\beta}\right\|_{\mathcal{F}_{i} \cup \mathcal{F}_{-}} \leq\left\||\beta \cdot n|^{\frac{1}{2}} \tilde{h}_{\mathcal{F}}[\nabla v]_{T}\right\|_{\mathcal{F}_{i} \cup \mathcal{F}_{-}}
$$

where here $[\nabla v]_{T}=\left.\nabla v\right|_{\kappa_{1}} \times n_{1}+\left.\nabla v\right|_{\kappa_{2}} \times n_{2}$ is the tangential jump of the gradient. It follows that an equivalent stabilization term is obtained penalizing the jumps of certain derivatives, leading to a term that is no more complicated or expensive to compute than in the standard case. The following analysis holds in this case also with minor modifications. 
3.1. Some Lemmas. The following lemma is basically only the integration by parts of the advection term.

Lemma 3.3. Let $v, w \in H^{1}(\mathcal{K})$, then

$$
\begin{aligned}
a(v, w) & =(\beta \cdot \nabla v+\mu v, w)_{\mathcal{K}}-\left(|\beta|[v]_{\beta},\{w\}\right)_{\mathcal{F}_{i} \cup \mathcal{F}_{-},}, \\
a(v, v) & =\left(\left(\mu-\frac{1}{2} \nabla \cdot \beta\right) v, v\right)_{\mathcal{K}}+\frac{1}{2}(|\beta \cdot n| v, v)_{\mathcal{F}_{e}} .
\end{aligned}
$$

Proof. The first equation is developed using integration by parts.. The second uses additionally the fact that $v=w$.

Corollary 3.4 (Coercivity). Let $v \in V_{h}^{p}$, then there exists a constant $c_{L}>0$, independent of $h$, such that

$$
c_{L} a\left(v_{h}, v_{h}\right) \geq\left(\left\|v_{h}\right\|_{\mathcal{K}}^{2}+\left\||\beta|^{\frac{1}{2}}\left[v_{h}\right]_{\beta}\right\|_{\mathcal{F}_{e}}^{2}\right) .
$$

This result follows immediately from (3.3) taking into account that $\left|n \cdot e_{\beta}\right| \leq 1$.

Lemma 3.5 (Consistency). Let $u \in H^{1}(\Omega)$ be the exact solution of problem (2.1) and let $u_{h}$ be the solution of (3.1), then

$$
a\left(u-u_{h}, v_{h}\right)+j\left(u-u_{h}, v_{h}\right)=0
$$

for all $v_{h} \in V_{h}^{p}$.

Proof. Since $u_{h}$ is the discrete solution it satisfies

$$
a\left(u_{h}, v_{h}\right)+j\left(u_{h}, v_{h}\right)=\left(f, v_{h}\right)_{\mathcal{K}} \quad \forall, v_{h} \in V_{h}^{p} .
$$

On the other hand since $u \in H^{1}(\Omega)$

$$
\left([u]_{\beta}, v_{h}\right)_{\mathcal{F}_{i}}=0 \quad \forall v_{h} \in V_{h}^{p},
$$

and thus $j\left(u, v_{h}\right)=0$ using additionally the boundary condition. Further applying Lemma 3.3 and the boundary condition yields

$$
\begin{aligned}
a\left(u, v_{h}\right) & =\left(\beta \cdot \nabla u+\mu u, v_{h}\right)_{\mathcal{K}}-\left(|\beta|[u]_{\beta},\left\{v_{h}\right\}\right)_{\mathcal{F}_{i} \cup \mathcal{F}_{-}} \\
& =\left(\beta \cdot \nabla u+\mu u, v_{h}\right)_{\mathcal{K}}-\left(|\beta|[u]_{\beta},\left\{v_{h}\right\}\right)_{\mathcal{F}_{-}} \\
& =\left(\beta \cdot \nabla u+\mu u, v_{h}\right)_{\mathcal{K}}=\left(f, v_{h}\right)_{\mathcal{K}} .
\end{aligned}
$$

\section{Convergence Analysis}

The triple norm is defined for all $v \in H^{1}(\mathcal{K})$ by

$$
\|v\|^{2}=\|v\|_{\mathcal{K}}^{2}+\left\|h^{\frac{1}{2}} \beta \cdot \nabla v\right\|_{\mathcal{K}}^{2}+\left\||\beta|^{\frac{1}{2}}[v]_{\beta}\right\|_{\mathcal{F}}^{2} .
$$

It allows to control the graph-norm as well as the solution jumps. First we develop some general results. The function $|\beta|_{\infty} \in W_{h}^{0}$ defined in section 3 has the following property

$$
\left\||\beta|-|\beta|_{\infty}\right\|_{L^{\infty}(F)} \leq c h_{F}\|\beta\|_{1, \infty, F}
$$

for every face $F \in \mathcal{F}$ since $\beta$ is Lipschitz continuous. Additionally define $\bar{\beta} \in V_{h}^{0}$ as the elementwise average of $\beta$, i.e.

$$
\left.\bar{\beta}\right|_{\kappa}=\frac{1}{|\kappa|} \int_{\kappa} \beta \quad \forall \kappa \in \mathcal{K} .
$$


Since $\beta$ is Lipschitz continuous we have

$$
\|\beta-\bar{\beta}\|_{L^{\infty}(\kappa)} \leq c h_{\kappa}\|\beta\|_{1, \infty, \kappa} .
$$

From this we deduce, that for any face $F=\partial \kappa \cup \partial \kappa^{\prime} \in \mathcal{F}$ we have

$$
\||\beta|-\{|\bar{\beta}|\}\|_{L^{\infty}(F)} \leq c\{\tilde{h}\}\|\beta\|_{1, \infty, \kappa \cup \kappa^{\prime}} \leq c h_{F}\|\beta\|_{1, \infty, \kappa \cup \kappa^{\prime}} .
$$

Proposition 4.1 (Inf-Sup Condition). Assume that $\beta \in[\operatorname{Lip}(\Omega)]^{2}$, then there exists a constant $c>0$, independent of the mesh size $h$, such that

$$
c\left|\left\|v_{h}\right\|\right| \leq \sup _{v_{h}^{\prime} \in V_{h}^{p}} \frac{a\left(v_{h}, v_{h}^{\prime}\right)+j\left(v_{h}, v_{h}^{\prime}\right)}{\left\|v_{h}^{\prime}\right\| \|} \quad \forall v_{h} \in V_{h}^{p} .
$$

Proof. For the proof of Proposition 4.1 we introduce the following two lemmas.

Lemma 4.1. For all $v_{h} \in V_{h}^{p}$ there exists $v_{h}^{\prime} \in V_{h}^{p}$ and $c>0$ such that

$$
c \mid\left\|v_{h}\right\|^{2} \leq a\left(v_{h}, v_{h}^{\prime}\right)+j\left(v_{h}, v_{h}^{\prime}\right) .
$$

Lemma 4.2. Fix $v_{h} \in V_{h}^{p}$ and let $v_{h}^{\prime} \in V_{h}^{p}$ be the function defined in Lemma 4.1, then there exists a constant $c>0$ such that

$$
\left\|v_{h}^{\prime}\right\||\leq c|\left\|v_{h}\right\| \mid
$$

Combining these two lemmas leads to the result. Indeed for all $v_{h} \in V_{h}^{p}$ there exists $v_{h}^{\prime} \in V_{h}^{p}$ and $c>0$ such that

$$
c\left\|v_{h}\right\| \leq \frac{a\left(v_{h}, v_{h}^{\prime}\right)+j\left(v_{h}, v_{h}^{\prime}\right)}{\left\|v_{h}^{\prime}\right\| \mid} .
$$

Proof of Lemma 4.1. Let us define

$$
\begin{aligned}
w_{h} & =\tilde{h} \bar{\beta} \cdot \nabla v_{h} \in V_{h}^{p}, \\
z_{h} & =\Pi_{h}\left(0, P_{l}\left[v_{h}\right]_{\beta}\right) \in V_{h}^{p},
\end{aligned}
$$

with $0 \leq l \leq\left\lfloor\frac{p+1}{3}\right\rfloor-1$. Let us first prove two preliminary results. Firstly, there exists a constants $c_{\beta}>0$ such that

$$
\left\|w_{h}\right\|_{\mathcal{K}} \leq c_{\beta} \min \left(\left\|\tilde{h} \beta \cdot \nabla v_{h}\right\|_{\mathcal{K}}+\left\|\tilde{h} v_{h}\right\|_{\mathcal{K}},\left\|v_{h}\right\|_{\mathcal{K}}\right)
$$

Indeed on one hand we have that

$$
\begin{aligned}
\left\|w_{h}\right\|_{\mathcal{K}} & \leq\left\|\tilde{h} \beta \cdot \nabla v_{h}\right\|_{\mathcal{K}}+\left\|\tilde{h}(\bar{\beta}-\beta) \cdot \nabla v_{h}\right\|_{\mathcal{K}} \leq\left\|\tilde{h} \beta \cdot \nabla v_{h}\right\|_{\mathcal{K}}+c\left\|\tilde{h}^{2} \nabla v_{h}\right\|_{\mathcal{K}} \\
& \leq\left\|\tilde{h} \beta \cdot \nabla v_{h}\right\|_{\mathcal{K}}+c\left\|\tilde{h} v_{h}\right\|_{\mathcal{K}},
\end{aligned}
$$

using (4.2) and Lemma 2.3, and on the other hand we note that by an inverse inequality

$$
\left\|w_{h}\right\|_{\mathcal{K}}=\left\|\tilde{h} \bar{\beta} \cdot \nabla v_{h}\right\|_{\mathcal{K}} \leq c\left\|v_{h}\right\|_{\mathcal{K}} .
$$

Secondly there exists a constant $c>0$ such that

$$
\begin{aligned}
\left\||\beta|_{\infty}^{\frac{1}{2}}\left\{z_{h}\right\}\right\|_{\mathcal{F}}^{2}+\left\||\beta|_{\infty}^{\frac{1}{2}}\left[z_{h}\right]_{\beta}\right\|_{\mathcal{F}}^{2} & \leq\left\||\beta|_{\infty}^{\frac{1}{2}} P_{l}\left[v_{h}\right]_{\beta}\right\|_{\mathcal{F}}^{2}+c\left\|v_{h}\right\|_{\mathcal{K}}^{2} \\
\left\|\tilde{h}_{\mathcal{F}}^{\frac{1}{2}}\left\{z_{h}\right\}\right\|_{\mathcal{F}}^{2}+\left\|\mid \tilde{h}_{\mathcal{F}}^{\frac{1}{2}}\left[z_{h}\right]_{\beta}\right\|_{\mathcal{F}}^{2} & \leq\left\|\tilde{h}_{\mathcal{F}}^{\frac{1}{2}} P_{l}\left[v_{h}\right]_{\beta}\right\|_{\mathcal{F}}^{2}+c\left\|v_{h}\right\|_{\mathcal{K}}^{2}
\end{aligned}
$$


Indeed let us fix an element $\kappa \in \mathcal{K}$ with boundary $\partial \kappa=F \cup F_{1} \cup F_{2}$. For $j=1,2$ let us note $c_{F}=\left.|\beta|_{\infty}\right|_{F}$ resp. $c_{F}=h_{F}$ and $c_{F_{j}}=\left.|\beta|_{\infty}\right|_{F_{j}}$ resp. $c_{F_{j}}=h_{F_{j}}$. Then using the local stability property of the projection (2.7) we develop

$$
\begin{aligned}
\left\|c_{F}^{\frac{1}{2}} z_{h}\right\|_{F}^{2} & \leq\left\|c_{F}^{\frac{1}{2}} z_{h}\right\|_{\partial \kappa}^{2} \leq c\left\|c_{F}^{\frac{1}{2}} P_{l}\left[v_{h}\right]_{\beta}\right\|_{\partial \kappa}^{2} \\
& \leq c\left(\left\|c_{F}^{\frac{1}{2}} P_{l}\left[v_{h}\right]_{\beta}\right\|_{F}^{2}+\left\|c_{F}^{\frac{1}{2}} P_{l}\left[v_{h}\right]_{\beta}\right\|_{F_{1}}^{2}+\left\|c_{F}^{\frac{1}{2}} P_{l}\left[v_{h}\right]_{\beta}\right\|_{F_{2}}^{2}\right)
\end{aligned}
$$

Then using a triangle inequality we may write for $j=1,2$

$$
\left\|c_{F}^{\frac{1}{2}} P_{l}\left[v_{h}\right]_{\beta}\right\|_{F_{j}}^{2} \leq\left\|c_{F_{j}}^{\frac{1}{2}} P_{l}\left[v_{h}\right]_{\beta}\right\|_{F_{j}}^{2}+\left\|\left(c_{F}^{\frac{1}{2}}-c_{F_{j}}^{\frac{1}{2}}\right) P_{l}\left[v_{h}\right]_{\beta}\right\|_{F_{j}}^{2} .
$$

The second term can further be developed using that $\beta \in[\operatorname{Lip}(\Omega)]^{2}$ resp. $\mid h_{F}-$ $h_{F_{j}} \mid \leq c h_{F_{j}}$ (shape-regularity), the stability of the projection (2.4) and the trace inequality

$$
\left\|\left(c_{F}^{\frac{1}{2}}-c_{F_{j}}^{\frac{1}{2}}\right) P_{l}\left[v_{h}\right]_{\beta}\right\|_{F_{j}}^{2} \leq c\left\|h_{F_{j}}^{\frac{1}{2}} P_{l}\left[v_{h}\right]_{\beta}\right\|_{F_{j}}^{2} \leq c\left\|h_{F_{j}}^{\frac{1}{2}}\left[v_{h}\right]_{\beta}\right\|_{F_{j}}^{2} \leq c\left\|v_{h}\right\|_{\kappa}^{2} .
$$

Thus

$$
\begin{aligned}
\left\|\left.\beta\right|_{\infty} ^{\frac{1}{2}} z_{h}\right\|_{F}^{2} & \leq\left\|\left.\beta\right|_{\infty} ^{\frac{1}{2}} P_{l}\left[v_{h}\right]_{\beta}\right\|_{\partial \kappa}^{2}+c\left\|v_{h}\right\|_{\kappa}^{2} \\
\left\|h_{F}^{\frac{1}{2}} z_{h}\right\|_{F}^{2} & \leq\left\|\tilde{h}_{\mathcal{F}}^{\frac{1}{2}} P_{l}\left[v_{h}\right]_{\beta}\right\|_{\partial \kappa}^{2}+c\left\|v_{h}\right\|_{\kappa}^{2}
\end{aligned}
$$

Cumulating (4.9) resp. (4.10) for all elements leads to (4.7) resp. (4.8). After these preliminary results, we prove the lemma in three steps.

Step 1 First we prove that there exists a constant $c_{w}>0$ such that

$$
\left\|\tilde{h}^{\frac{1}{2}} \beta \cdot \nabla v_{h}\right\|_{\mathcal{K}}^{2} \leq a\left(v_{h}, c_{w} v_{h}+4 w_{h}\right)+j\left(v_{h}, c_{w} v_{h}+4 w_{h}\right)+c_{w}\left\||\beta|^{\frac{1}{2}}\left[v_{h}\right]_{\beta}\right\|_{\mathcal{F}_{i}}^{2} .
$$

By the definition of the bilinear form $a(\cdot, \cdot)$ we obtain

$$
\begin{aligned}
\left\|\tilde{h}^{\frac{1}{2}} \beta \cdot \nabla v_{h}\right\|_{\mathcal{K}}^{2}= & a\left(v_{h}, w_{h}\right)+\left(\beta \cdot \nabla v_{h}, \tilde{h}(\beta-\bar{\beta}) \cdot \nabla v_{h}\right)_{\mathcal{K}}-\left(\mu v_{h}, w_{h}\right)_{\mathcal{K}} \\
& +\left(|\beta|\left[v_{h}\right]_{\beta},\left\{w_{h}\right\}\right)_{\mathcal{F}_{i} \cup \mathcal{F}_{-}}=a\left(v_{h}, w_{h}\right)+\mathcal{I}_{1}+\mathcal{I}_{2}+\mathcal{I}_{3} .
\end{aligned}
$$

Then develop each term. For the first we use relation (4.2):

$$
\begin{aligned}
\left|\mathcal{I}_{1}\right| & \leq c\left\|\tilde{h}^{\frac{1}{2}} \beta \cdot \nabla v_{h}\right\|_{\mathcal{K}}\left\|\tilde{h}^{\frac{3}{2}} \nabla v_{h}\right\|_{\mathcal{K}} \leq c\left\|\tilde{h}^{\frac{1}{2}} \beta \cdot \nabla v_{h}\right\|_{\mathcal{K}}\left\|\tilde{h}^{\frac{1}{2}} v_{h}\right\|_{\mathcal{K}} \\
& \leq c\left\|\tilde{h}^{\frac{1}{2}} v_{h}\right\|_{\mathcal{K}}^{2}+\delta_{1}\left\|\tilde{h}^{\frac{1}{2}} \beta \cdot \nabla v_{h}\right\|_{\mathcal{K}}^{2}
\end{aligned}
$$

where $\delta_{1}>0$ is an arbitrary constant. For the second one we use (4.6)

$$
\left|\mathcal{I}_{2}\right| \leq c\left\|v_{h}\right\|_{\mathcal{K}}\left\|w_{h}\right\|_{\mathcal{K}} \leq c\left\|v_{h}\right\|_{\mathcal{K}}^{2} .
$$

To get an upper bound of the last term we use the trace inequality, Lemma 2.2 , followed by (4.6):

$$
\begin{aligned}
& \left|\mathcal{I}_{3}\right| \leq c\left\||\beta|^{\frac{1}{2}}\left[v_{h}\right]_{\beta}\right\|_{\mathcal{F}_{i} \cup \mathcal{F}_{-}}^{2}+\delta_{2}\left\|\left\{w_{h}\right\}\right\|_{\mathcal{F}_{i} \cup \mathcal{F}_{-}}^{2} \leq c\left\||\beta|^{\frac{1}{2}}\left[v_{h}\right]_{\beta}\right\|_{\mathcal{F}}^{2}+\delta_{2} c_{T}\left\|\tilde{h}^{-\frac{1}{2}} w_{h}\right\|_{\mathcal{K}}^{2} \\
& (4.14) \leq c\left(\left\||\beta|^{\frac{1}{2}}\left[v_{h}\right]_{\beta}\right\|_{\mathcal{F}}^{2}+\left\|v_{h}\right\|_{\mathcal{K}}^{2}\right)+\delta_{2} c_{T} c_{\beta}\left\|\tilde{h}^{\frac{1}{2}} \beta \cdot \nabla v_{h}\right\|_{\mathcal{K}}^{2}
\end{aligned}
$$

where $\delta_{2}>0$ is an arbitrary constant. Respect all bounds (4.12), (4.13), (4.14) and choose $\delta_{1}=\frac{1}{4}, \delta_{2}=\frac{1}{4 c_{T} c_{\beta}}$. Then injecting these bounds in (4.11) yields

$$
\frac{1}{2}\left\|\tilde{h}^{\frac{1}{2}} \beta \cdot \nabla v_{h}\right\|_{\mathcal{K}}^{2} \leq a\left(v_{h}, w_{h}\right)+c\left(\left\|v_{h}\right\|_{\mathcal{K}}^{2}+\left\||\beta|^{\frac{1}{2}}\left[v_{h}\right]_{\beta}\right\|_{\mathcal{F}}^{2}\right) .
$$


We now consider the penalization term and use the trace inequality, Lemma 2.2, and (4.6) to develop

$$
\begin{aligned}
-j\left(v_{h}, w_{h}\right) & \leq c j\left(v_{h}, v_{h}\right)^{\frac{1}{2}}\left\|\left(I-P_{l}\right)\left[w_{h}\right]_{\beta}\right\|_{\mathcal{F}} \leq c j\left(v_{h}, v_{h}\right)+\delta_{2}\left\|\left[w_{h}\right]_{\beta}\right\|_{\mathcal{F}}^{2} \\
& \leq c j\left(v_{h}, v_{h}\right)+\delta_{2} c_{T}\left\|\tilde{h}^{-\frac{1}{2}} w_{h}\right\|_{\mathcal{K}}^{2} \\
& \leq c j\left(v_{h}, v_{h}\right)+\delta_{2} c_{T} c_{\beta}\left\|\tilde{h}^{\frac{1}{2}} \beta \cdot \nabla v_{h}\right\|_{\mathcal{K}}^{2}+\delta_{2} c_{T} c_{\beta}\left\|\tilde{h}^{\frac{1}{2}} v_{h}\right\|_{\mathcal{K}}^{2} .
\end{aligned}
$$

Since $\delta_{2}=\frac{1}{4 c_{T} c_{\beta}}$ we get

$$
0 \leq j\left(v_{h}, c v_{h}+w_{h}\right)+\frac{1}{4}\left\|\tilde{h}^{\frac{1}{2}} \beta \cdot \nabla v_{h}\right\|_{\mathcal{K}}^{2}+\frac{1}{4}\left\|\tilde{h}^{\frac{1}{2}} v_{h}\right\|_{\mathcal{K}}^{2} .
$$

Combining (4.15), (4.16) and the coercivity of Lemma 3.4 leads to

$$
\begin{aligned}
\frac{1}{4}\left\|h^{\frac{1}{2}} \beta \cdot \nabla v_{h}\right\|_{\mathcal{K}}^{2} & \leq a\left(v_{h}, w_{h}\right)+j\left(v_{h}, c v_{h}+w_{h}\right)+c\left(\left\|v_{h}\right\|_{\mathcal{K}}^{2}+\left\||\beta|^{\frac{1}{2}}\left[v_{h}\right]_{\beta}\right\|_{\mathcal{F}}^{2}\right) \\
& \leq a\left(v_{h}, c v_{h}+w_{h}\right)+j\left(v_{h}, c v_{h}+w_{h}\right)+c\left\||\beta|^{\frac{1}{2}}\left[v_{h}\right]_{\beta}\right\|_{\mathcal{F}_{i}}^{2} .
\end{aligned}
$$

Step 2 Then we prove that there exists a constant $c_{z}>0$ such that

$$
\left\||\beta|^{\frac{1}{2}}\left[v_{h}\right]_{\beta}\right\|_{\mathcal{F}_{i}}^{2} \leq a\left(v_{h}, c_{z} v_{h}-4 z_{h}\right)+j\left(v_{h}, c_{z} v_{h}-4 z_{h}\right) .
$$

By Lemma 3.3 and the property of the projection, relation (2.5), we obtain

$$
\begin{aligned}
a\left(v_{h}, z_{h}\right)= & \left(\beta \cdot \nabla v_{h}+\mu v_{h}, z_{h}\right)_{\mathcal{K}}-\left(|\beta|\left[v_{h}\right]_{\beta},\left\{z_{h}\right\}\right)_{\mathcal{F}_{i} \cup \mathcal{F}_{-}} \\
= & \left((\beta-\bar{\beta}) \cdot \nabla v_{h}, z_{h}\right)_{\mathcal{K}}+\left(\mu v_{h}, z_{h}\right)_{\mathcal{K}}-\left(|\beta|_{\infty}\left[v_{h}\right]_{\beta},\left\{z_{h}\right\}\right)_{\mathcal{F}_{i} \cup \mathcal{F}_{-}} \\
& -\left(\left[v_{h}\right]_{\beta},\left(|\beta|-|\beta|_{\infty}\right)\left\{z_{h}\right\}\right)_{\mathcal{F}_{i} \cup \mathcal{F}_{-}} \\
= & \left((\beta-\bar{\beta}) \cdot \nabla v_{h}, z_{h}\right)_{\mathcal{K}}+\left(\mu v_{h}, z_{h}\right)_{\mathcal{K}}-\left(|\beta|_{\infty} P_{l}\left[v_{h}\right]_{\beta},\left\{z_{h}\right\}\right)_{\mathcal{F}_{i} \cup \mathcal{F}_{-}} \\
& -\left(|\beta|_{\infty}\left(I-P_{l}\right)\left[v_{h}\right]_{\beta},\left\{z_{h}\right\}\right)_{\mathcal{F}_{i} \cup \mathcal{F}_{-}}-\left(\left[v_{h}\right]_{\beta},\left(|\beta|-|\beta|_{\infty}\right)\left\{z_{h}\right\}\right)_{\mathcal{F}_{i} \cup \mathcal{F}_{-}}
\end{aligned}
$$

Thus by (2.6):

$$
\begin{aligned}
\left\||\beta|_{\infty}^{\frac{1}{2}} P_{l}\left[v_{h}\right]_{\beta}\right\|_{\mathcal{F}_{i} \leq} \leq & -a\left(v_{h}, z_{h}\right)+\left((\beta-\bar{\beta}) \cdot \nabla v_{h}, z_{h}\right)_{\mathcal{K}}+\left(\mu v_{h}, z_{h}\right)_{\mathcal{K}} \\
& -\left(|\beta|_{\infty}\left(I-P_{l}\right)\left[v_{h}\right]_{\beta},\left\{z_{h}\right\}\right)_{\mathcal{F}_{i} \cup \mathcal{F}_{-}} \\
& -\left(\left[v_{h}\right]_{\beta},\left(|\beta|-|\beta|_{\infty}\right)\left\{z_{h}\right\}\right)_{\mathcal{F}_{i} \cup \mathcal{F}_{-}} \\
= & -a\left(v_{h}, z_{h}\right)+\mathcal{I}_{1}+\mathcal{I}_{2}+\mathcal{I}_{3}+\mathcal{I}_{4}
\end{aligned}
$$

For the first term $\mathcal{I}_{1}$, we apply relation (4.2), the inverse inequality of Lemma 2.3, property (2.9), relation (2.4) and the trace inequality of Lemma 2.2:

$$
\begin{aligned}
\left|\mathcal{I}_{1}\right| & \leq c\left\|\tilde{h} \nabla v_{h}\right\|_{\mathcal{K}}\left\|z_{h}\right\|_{\mathcal{K}} \leq c\left\|v_{h}\right\|_{\mathcal{K}}\left\|\tilde{h}_{\mathcal{F}}^{\frac{1}{2}} P_{l}\left[v_{h}\right]_{\beta}\right\|_{\mathcal{F}} \leq c\left\|v_{h}\right\|_{\mathcal{K}}\left\|\tilde{h}_{\mathcal{F}}^{\frac{1}{2}}\left[v_{h}\right]_{\beta}\right\|_{\mathcal{F}} \\
& \leq c\left\|v_{h}\right\|_{\mathcal{K}}^{2}
\end{aligned}
$$

Use property (2.9), relation (2.4) and the trace inequality, Lemma 2.2, for the second term

$$
\left|\mathcal{I}_{2}\right| \leq c\left\|v_{h}\right\|_{\mathcal{K}}\left\|z_{h}\right\|_{\mathcal{K}} \leq c\left\|v_{h}\right\|_{\mathcal{K}}\left\|\tilde{h}_{\mathcal{F}}^{\frac{1}{2}} P_{l}\left[v_{h}\right]_{\beta}\right\|_{\mathcal{F}} \leq c\left\|v_{h}\right\|_{\mathcal{K}}\left\|\tilde{h}_{\mathcal{F}}^{\frac{1}{2}}\left[v_{h}\right]_{\beta}\right\|_{\mathcal{F}} \leq c\left\|v_{h}\right\|_{\mathcal{K}}^{2}
$$
and property (4.7) for the third one

$$
\begin{aligned}
\left|\mathcal{I}_{3}\right| & \leq c j\left(v_{h}, v_{h}\right)^{\frac{1}{2}}\left\||\beta|_{\infty}^{\frac{1}{2}}\left\{z_{h}\right\}\right\|_{\mathcal{F}} \leq c j\left(v_{h}, v_{h}\right)+\delta_{3}\left\||\beta|_{\infty}^{\frac{1}{2}}\left\{z_{h}\right\}\right\|_{\mathcal{F}}^{2} \\
& \leq c\left(j\left(v_{h}, v_{h}\right)+\left\|v_{h}\right\|_{\mathcal{K}}^{2}\right)+\delta_{3}\left\||\beta|_{\infty}^{\frac{1}{2}} P_{l}\left[v_{h}\right]_{\beta}\right\|_{\mathcal{F}}^{2} .
\end{aligned}
$$


Then for the last term we may write

$$
\begin{aligned}
& \left|\mathcal{I}_{4}\right| \leq c\left\|\tilde{h}_{\mathcal{F}}^{\frac{1}{2}}\left[v_{h}\right]_{\beta}\right\|_{\mathcal{F}}\left\|\tilde{h}_{\mathcal{F}}^{\frac{1}{2}}\left\{z_{h}\right\}\right\|_{\mathcal{F}} \leq c\left\|\tilde{h}_{\mathcal{F}}^{\frac{1}{2}}\left[v_{h}\right]_{\beta}\right\|_{\mathcal{F}}\left(\left\|\tilde{h}_{\mathcal{F}}^{\frac{1}{2}} P_{l}\left[v_{h}\right]_{\beta}\right\|_{\mathcal{F}}+\left\|v_{h}\right\|_{\mathcal{K}}\right) \\
& \leq c\left\|\tilde{h}_{\mathcal{F}}^{\frac{1}{2}}\left[v_{h}\right]_{\beta}\right\|_{\mathcal{F}}\left(\left\|\tilde{h}_{\mathcal{F}}^{\frac{1}{2}}\left[v_{h}\right]_{\beta}\right\|_{\mathcal{F}}+\left\|v_{h}\right\|_{\mathcal{K}}\right) \leq\left\|v_{h}\right\|_{\mathcal{K}}^{2}
\end{aligned}
$$

using (4.1), property (4.8), relation (2.4) and the trace inequality, Lemma 2.2. Thus respecting all bounds $(4.18),(4.19),(4.20)$ and $(4.21)$ in $(4.17)$ with $\delta_{3}=\frac{1}{2}$ leads to

$$
\frac{1}{2}\left\||\beta|_{\infty}^{\frac{1}{2}} P_{l}\left[v_{h}\right]_{\beta}\right\|_{\mathcal{F}_{i}}^{2} \leq-a\left(v_{h}, z_{h}\right)+c\left(\left\|v_{h}\right\|_{\mathcal{K}}^{2}+\left\||\beta|_{\infty}^{\frac{1}{2}}\left[v_{h}\right]_{\beta}\right\|_{\mathcal{F}_{e}}^{2}\right)+c j\left(v_{h}, v_{h}\right)
$$

Then use (4.7) to develop

$$
\begin{aligned}
& j\left(v_{h}, z_{h}\right) \\
& \quad \leq j\left(v_{h}, v_{h}\right)^{\frac{1}{2}}\left\||\beta|_{\infty}^{\frac{1}{2}}\left(I-P_{l}\right)\left[z_{h}\right]_{\beta}\right\|_{\mathcal{F}} \leq j\left(v_{h}, v_{h}\right)^{\frac{1}{2}}\left\||\beta|_{\infty}^{\frac{1}{2}}\left[z_{h}\right]_{\beta}\right\|_{\mathcal{F}} \\
& \quad \leq c j\left(v_{h}, v_{h}\right)+\delta_{4}\left\||\beta|_{\infty}^{\frac{1}{2}}\left[z_{h}\right]_{\beta}\right\|_{\mathcal{F}}^{2} \leq c\left(j\left(v_{h}, v_{h}\right)+\left\|v_{h}\right\|_{\mathcal{K}}^{2}\right)+\delta_{4}\left\||\beta|_{\infty}^{\frac{1}{2}} P_{l}\left[v_{h}\right]_{\beta}\right\|_{\mathcal{F}}^{2} .
\end{aligned}
$$

Choose $\delta_{4}=\frac{1}{4}$ and therefore

$\frac{1}{4}\left\||\beta|^{\frac{1}{2}} P_{l}\left[v_{h}\right]_{\beta}\right\|_{\mathcal{F}_{i}}^{2}$

$$
\leq \frac{1}{4}\left\||\beta|_{\infty}^{\frac{1}{2}} P_{l}\left[v_{h}\right]_{\beta}\right\|_{\mathcal{F}_{i}}^{2} \leq-a\left(v_{h}, z_{h}\right)+c\left(\left\|v_{h}\right\|_{\mathcal{K}}^{2}+\left\||\beta|_{\infty}^{\frac{1}{2}}\left[v_{h}\right]_{\beta}\right\|_{\mathcal{F}_{e}}^{2}\right)+j\left(v_{h}, c v_{h}-z_{h}\right) .
$$

Additionally observe that

$$
\begin{aligned}
\left\||\beta|_{\infty}^{\frac{1}{2}}\left[v_{h}\right]_{\beta}\right\|_{\mathcal{F}_{e}}^{2} & \leq\left\||\beta|^{\frac{1}{2}}\left[v_{h}\right]_{\beta}\right\|_{\mathcal{F}_{e}}^{2}+\left\|\left(|\beta|_{\infty}-|\beta|\right)^{\frac{1}{2}}\left[v_{h}\right]_{\beta}\right\|_{\mathcal{F}_{e}}^{2} \\
& \leq\left\||\beta|^{\frac{1}{2}}\left[v_{h}\right]_{\beta}\right\|_{\mathcal{F}_{e}}^{2}+c\left\|\tilde{h}_{\mathcal{F}}^{\frac{1}{2}}\left[v_{h}\right]_{\beta}\right\|_{\mathcal{F}_{e}}^{2} \leq\left\||\beta|^{\frac{1}{2}}\left[v_{h}\right]_{\beta}\right\|_{\mathcal{F}_{e}}^{2}+c\left\|v_{h}\right\|_{\mathcal{K}}^{2}
\end{aligned}
$$

using (4.1) and the trace inequality. Thus we have the following upper bound for the solution jumps

$$
\frac{1}{4}\left\||\beta|^{\frac{1}{2}} P_{l}\left[v_{h}\right]_{\beta}\right\|_{\mathcal{F}_{i}}^{2} \leq-a\left(v_{h}, z_{h}\right)+c\left(\left\|v_{h}\right\|_{\mathcal{K}}^{2}+\left\||\beta|^{\frac{1}{2}}\left[v_{h}\right]_{\beta}\right\|_{\mathcal{F}_{e}}^{2}\right)+j\left(v_{h}, c v_{h}-z_{h}\right) .
$$

Now apply the coercivity, Corollary 3.4, to conclude

$$
\frac{1}{4}\left\||\beta|^{\frac{1}{2}}\left[v_{h}\right]_{\beta}\right\|_{\mathcal{F}_{i}}^{2} \leq a\left(v_{h}, c v_{h}-z_{h}\right)+j\left(v_{h}, c v_{h}-z_{h}\right) .
$$

Step 3 Finally combining the coercivity of Corollary 3.4, Step 1 and Step 2, we may write

$$
\begin{aligned}
\|\| v_{h} \|^{2} & =\left\|v_{h}\right\|_{\mathcal{K}}^{2}+\left\|\tilde{h}^{\frac{1}{2}} \beta \cdot \nabla v_{h}\right\|_{\mathcal{K}}^{2}+\left\||\beta|^{\frac{1}{2}}\left[v_{h}\right]_{\beta}\right\|_{\mathcal{F}}^{2} \\
& \leq a\left(v_{h}, c_{L} v_{h}\right)+\left\|\tilde{h}^{\frac{1}{2}} \beta \cdot \nabla v_{h}\right\|_{\mathcal{K}}^{2}+\left\||\beta|^{\frac{1}{2}}\left[v_{h}\right]_{\beta}\right\|_{\mathcal{F}_{i}}^{2} \\
& \leq a\left(v_{h},\left(c_{L}+c_{w}\right) v_{h}+4 w_{h}\right)+j\left(v_{h}, c_{w} v_{h}+4 w_{h}\right)+\left(1+c_{w}\right)\left\||\beta|^{\frac{1}{2}}\left[v_{h}\right]_{\beta}\right\|_{\mathcal{F}_{i}}^{2} \\
& \leq a\left(v_{h}, v_{h}^{\prime}\right)+j\left(v_{h}, v_{h}^{\prime}\right)
\end{aligned}
$$

with $v_{h}^{\prime}=\left(c_{L}+c_{w}+\left(1+c_{w}\right) c_{z}\right) v_{h}+4 w_{h}-4\left(1+c_{w}\right) z_{h}=c_{1} v_{h}+c_{2} w_{h}-c_{3} z_{h}$.

Proof of Lemma 4.2. By definition of the triple norm:

$$
\left\|v_{h}^{\prime}\right\|^{2}=\left\|v_{h}^{\prime}\right\|_{\mathcal{K}}^{2}+\left\|\tilde{h}^{\frac{1}{2}} \beta \cdot \nabla v_{h}^{\prime}\right\|_{\mathcal{K}}^{2}+\left\||\beta|^{\frac{1}{2}}\left[v_{h}^{\prime}\right]_{\beta}\right\|_{\mathcal{F}}^{2}
$$

Recall the definition for $v_{h}^{\prime}=c_{1} v_{h}+c_{2} w_{h}-c_{3} z_{h}$ and develop the first term of (4.23):

$$
\left\|v_{h}^{\prime}\right\|_{\mathcal{K}}^{2} \leq c\left(c_{1}^{2}\left\|v_{h}\right\|_{\mathcal{K}}^{2}+c_{2}^{2}\left\|w_{h}\right\|_{\mathcal{K}}^{2}+c_{3}^{2}\left\|z_{h}\right\|_{\mathcal{K}}^{2}\right)
$$


For the second term of (4.24) use (4.6):

$$
\left\|w_{h}\right\|_{\mathcal{K}}^{2} \leq\left\|\tilde{h}^{\frac{1}{2}} \beta \cdot \nabla v_{h}\right\|_{\mathcal{K}}^{2}+c\left\|v_{h}\right\|_{\mathcal{K}}^{2}
$$

then use property (2.9) for the third term of (4.24):

$$
\left\|z_{h}\right\|_{\mathcal{K}}^{2} \leq c\left\|\tilde{h}_{\mathcal{F}}^{\frac{1}{2}} P_{l}\left[v_{h}\right]_{\beta}\right\|_{\mathcal{F}}^{2} \leq c\left\|\tilde{h}_{\mathcal{F}}^{\frac{1}{2}}\left[v_{h}\right]_{\beta}\right\|_{\mathcal{F}}^{2} \leq c\left\|v_{h}\right\|_{\mathcal{K}}^{2} .
$$

Thus

$$
\left\|v_{h}^{\prime}\right\|_{\mathcal{K}}^{2} \leq c \mid\left\|v_{h}\right\|^{2} .
$$

Now we develop the second term of (4.23):

$$
\left\|\tilde{h}^{\frac{1}{2}} \beta \cdot \nabla v_{h}^{\prime}\right\|_{\mathcal{K}}^{2} \leq c\left(c_{1}^{2}\left\|\tilde{h}^{\frac{1}{2}} \beta \cdot \nabla v_{h}\right\|_{\mathcal{K}}^{2}+c_{2}^{2}\left\|\tilde{h}^{\frac{1}{2}} \beta \cdot \nabla w_{h}\right\|_{\mathcal{K}}^{2}+c_{3}^{2}\left\|\tilde{h}^{\frac{1}{2}} \beta \cdot \nabla z_{h}\right\|_{\mathcal{K}}^{2}\right) .
$$

For the second term of (4.26), the inverse inequality, Lemma 2.3, and (4.6) is used:

$$
\left\|\tilde{h}^{\frac{1}{2}} \beta \cdot \nabla w_{h}\right\|_{\mathcal{K}}^{2} \leq c\left\|\tilde{h}^{-\frac{1}{2}} w_{h}\right\|_{\mathcal{K}}^{2} \leq c\left\|\tilde{h}^{\frac{1}{2}} \beta \cdot \nabla v_{h}\right\|_{\mathcal{K}}^{2}+c\left\|v_{h}\right\|_{\mathcal{K}}^{2} .
$$

Before the third term of (4.26) can be bounded we use (4.3) to develop

$$
\begin{aligned}
\left\|\{|\bar{\beta}|\}\left[v_{h}\right]_{\beta}\right\|_{\mathcal{F}}^{2} & =\left\|(\{|\bar{\beta}|\}-|\beta|)^{\frac{1}{2}}\left[v_{h}\right]_{\beta}\right\|_{\mathcal{F}}^{2}+\left\||\beta|^{\frac{1}{2}}\left[v_{h}\right]_{\beta}\right\|_{\mathcal{F}}^{2} \\
& \leq c\left\|\tilde{h}_{\mathcal{F}}^{\frac{1}{2}}\left[v_{h}\right]_{\beta}\right\|_{\mathcal{F}}^{2}+\left\||\beta|^{\frac{1}{2}}\left[v_{h}\right]_{\beta}\right\|_{\mathcal{F}}^{2} \leq c\left\|v_{h}\right\|_{\mathcal{K}}^{2}+\left\||\beta|^{\frac{1}{2}}\left[v_{h}\right]_{\beta}\right\|_{\mathcal{F}}^{2} .
\end{aligned}
$$

Thus

$$
\begin{aligned}
& \left\|\tilde{h}^{\frac{1}{2}} \beta \cdot \nabla z_{h}\right\|_{\mathcal{K}}^{2} \\
& \quad \leq c\left(\left\|\tilde{h}^{\frac{1}{2}} \bar{\beta} \cdot \nabla z_{h}\right\|_{\mathcal{K}}^{2}+\left\|\tilde{h}^{\frac{1}{2}}(\beta-\bar{\beta}) \cdot \nabla z_{h}\right\|_{\mathcal{K}}^{2}\right) \leq c\left(\left\|\tilde{h}^{\frac{1}{2}}|\bar{\beta}| \nabla z_{h}\right\|_{\mathcal{K}}^{2}+\left\|\tilde{h}^{\frac{3}{2}} \nabla z_{h}\right\|_{\mathcal{K}}^{2}\right) \\
& \quad \leq c\left(\left\|\tilde{h}^{-\frac{1}{2}}|\bar{\beta}| z_{h}\right\|_{\mathcal{K}}^{2}+\left\|\tilde{h}^{\frac{1}{2}} z_{h}\right\|_{\mathcal{K}}^{2}\right) \leq c\left(\left\|\{|\bar{\beta}|\} P_{l}\left[v_{h}\right]_{\beta}\right\|_{\mathcal{F}}^{2}+\left\|\tilde{h}_{\mathcal{F}} P_{l}\left[v_{h}\right]_{\beta}\right\|_{\mathcal{F}}^{2}\right) \\
& \quad \leq c\left(\left\|\{|\bar{\beta}|\}\left[v_{h}\right]_{\beta}\right\|_{\mathcal{F}}^{2}+\left\|\tilde{h}_{\mathcal{F}}\left[v_{h}\right]_{\beta}\right\|_{\mathcal{F}}^{2}\right) \leq c\left(\left\||\beta|^{\frac{1}{2}}\left[v_{h}\right]_{\beta}\right\|_{\mathcal{F}}^{2}+\left\|v_{h}\right\|_{\mathcal{K}}^{2}\right)
\end{aligned}
$$

where we have applied (4.2) followed by Lemma 2.3, property (2.10), (2.4) and (4.27). It follows that

$$
\left\|\tilde{h}^{\frac{1}{2}} \beta \cdot \nabla v_{h}^{\prime}\right\|_{\mathcal{K}}^{2} \leq c\|\| v_{h} \|^{2} .
$$

Finally the third term of (4.23) is developed

$$
\left\||\beta|^{\frac{1}{2}}\left[v_{h}^{\prime}\right]_{\beta}\right\|_{\mathcal{F}}^{2} \leq c\left(c_{1}^{2}\left\||\beta|^{\frac{1}{2}}\left[v_{h}\right]_{\beta}\right\|_{\mathcal{F}}^{2}+c_{2}^{2}\left\||\beta|^{\frac{1}{2}}\left[w_{h}\right]_{\beta}\right\|_{\mathcal{F}}^{2}+c_{2}^{2}\left\|\tilde{h}|\beta|^{\frac{1}{2}}\left[z_{h}\right]_{\beta}\right\|_{\mathcal{F}}^{2}\right) .
$$

Use the trace inequality of Lemma 2.2, (4.6) and Lemma 2.3 for the second part of (4.29):

$$
\left\||\beta|^{\frac{1}{2}}\left[w_{h}\right]_{\beta}\right\|_{\mathcal{F}}^{2} \leq c\left\|\left[w_{h}\right]_{\beta}\right\|_{\mathcal{F}}^{2} \leq c\left\|\tilde{h}^{-\frac{1}{2}} w_{h}\right\|_{\mathcal{K}}^{2} \leq c\left\|\tilde{h}^{\frac{1}{2}} \beta \cdot \nabla v_{h}\right\|_{\mathcal{K}}^{2}+c\left\|v_{h}\right\|_{\mathcal{K}}^{2},
$$

and (4.7), (2.4), (4.1) and the trace inequality, Lemma 2.2, for the third term of (4.29):

$$
\begin{aligned}
& \left\||\beta|^{\frac{1}{2}}\left[z_{h}\right]_{\beta}\right\|_{\mathcal{F}}^{2} \\
& \quad \leq\left\|\left.|| \beta\right|_{\infty} ^{\frac{1}{2}}\left[z_{h}\right]_{\beta}\right\|_{\mathcal{F}}^{2} \leq\left\||\beta|_{\infty}^{\frac{1}{2}} P_{l}\left[v_{h}\right]_{\beta}\right\|_{\mathcal{F}}^{2}+c\left\|v_{h}\right\|_{\mathcal{K}}^{2} \leq c\left(\left\||\beta|_{\infty}^{\frac{1}{2}}\left[v_{h}\right]_{\beta}\right\|_{\mathcal{F}}^{2}+\left\|v_{h}\right\|_{\mathcal{K}}^{2}\right) \\
& \quad \leq c\left(\left\||\beta|^{\frac{1}{2}}\left[v_{h}\right]_{\beta}\right\|_{\mathcal{F}}^{2}+c\left\|\left(|\beta|_{\infty}-|\beta|\right)^{\frac{1}{2}}\left[v_{h}\right]_{\beta}\right\|_{\mathcal{F}}^{2}+\left\|v_{h}\right\|_{\mathcal{K}}^{2}\right) \\
& \quad \leq c\left(\left\||\beta|^{\frac{1}{2}}\left[v_{h}\right]_{\beta}\right\|_{\mathcal{F}}^{2}+c\left\|\tilde{h}_{\mathcal{F}}^{\frac{1}{2}}\left[v_{h}\right]_{\beta}\right\|_{\mathcal{F}}^{2}+\left\|v_{h}\right\|_{\mathcal{K}}^{2}\right) \leq c\left(\left\|\left.|| \beta\right|^{\frac{1}{2}}\left[v_{h}\right]_{\beta}\right\|_{\mathcal{F}}^{2}+c\left\|v_{h}\right\|_{\mathcal{K}}^{2}\right) .
\end{aligned}
$$


Thus

$$
\left\||\beta|^{\frac{1}{2}}\left[v_{h}^{\prime}\right]_{\beta}\right\|_{\mathcal{F}}^{2} \leq c \mid\left\|v_{h}\right\|^{2} .
$$

Respecting all bounds (4.25), (4.28) and (4.30) leads to the result.

Let $u$ denote the exact solution of (2.1) and $u_{h}$ the solution of (3.1), then define

$$
\eta=u-P_{h} u \quad \text { and } \quad \xi_{h}=u_{h}-P_{h} u,
$$

where $P_{h}$ denotes the elementwise $L^{2}$-projection of order $p$. To prove continuity of the bilinear form $a(\cdot, \cdot)+j(\cdot, \cdot)$ we need to define an auxiliary norm:

$$
\|] v\left\|^{2}=\right\| \tilde{h}^{-\frac{1}{2}} v\left\|_{\mathcal{K}}^{2}+\right\|[v]_{\beta}\left\|_{\mathcal{F}}^{2}+\right\|\{v\} \|_{\mathcal{F}}^{2} \quad \forall v \in H^{1}(\mathcal{K}) .
$$

Proposition 4.2 (Continuity). Let $\eta$ and $\xi_{h}$ be defined by (4.31). Then there exists a constant $c>0$ such that

$$
\left.a\left(\eta, \xi_{h}\right)+j\left(\eta, \xi_{h}\right) \leq c \mid\right] \eta\left[\left|\left\|\xi_{h}\right\|\right| .\right.
$$

Proof. Develop the first part

$$
a\left(\eta, \xi_{h}\right)=-\left(\eta, \beta \cdot \nabla \xi_{h}\right)_{\mathcal{K}}+\left(|\beta|\{\eta\},\left[\xi_{h}\right]_{\beta}\right)_{\mathcal{F}_{i} \cup \mathcal{F}_{+}}+\left(\mu \eta, \xi_{h}\right)_{\mathcal{K}}
$$

and treat these three terms separately. We conclude immediately that the first term of (4.32) can be bounded using a Cauchy-Schwarz inequality,

$$
\left.-\left(\eta, \beta \cdot \nabla \xi_{h}\right)_{\mathcal{K}} \leq\left\|\tilde{h}^{-\frac{1}{2}} \eta\right\|_{\mathcal{K}}\left\|\tilde{h}^{\frac{1}{2}} \beta \cdot \nabla \xi_{h}\right\|_{\mathcal{K}} \leq \|\right] \eta\left[\|\mid\| \xi_{h} \| .\right.
$$

And similarly for

$$
\left.\left(|\beta|\{\eta\},\left[\xi_{h}\right]_{\beta}\right)_{\mathcal{F}_{i} \cup \mathcal{F}_{-}} \leq\|\{\eta\}\|_{\mathcal{F}}\left\||\beta|^{\frac{1}{2}}\left[\xi_{h}\right]_{\beta}\right\|_{\mathcal{F}} \leq \mid\right] \eta\left[\mid\left\|\xi_{h}\right\| \|,\right.
$$

and

$$
\left.\left(\mu \eta, \xi_{h}\right)_{\mathcal{K}} \leq c\|\eta\|_{\mathcal{K}}\left\|\xi_{h}\right\|_{\mathcal{K}} \leq \mid\right] \eta\left[\mid\left\|\xi_{h}\right\| \| .\right.
$$

Finally use the Cauchy-Schwarz inequality and the stability result (2.4) for the last term

$$
\left.j\left(\eta, \xi_{h}\right) \leq j(\eta, \eta)^{\frac{1}{2}} j\left(\xi_{h}, \xi_{h}\right)^{\frac{1}{2}} \leq \mid\right] \eta\left[\left|\left\|\left|\xi_{h} \|\right| .\right.\right.\right.
$$

Respecting all bounds yields

$$
\left.a\left(\eta, \xi_{h}\right)+j\left(\eta, \xi_{h}\right) \leq c \mid\right] \eta\left[||\left\|\xi_{h}\right\| \mid .\right.
$$

Proposition 4.3 (Approximability). Assume that the exact solution of (2.1) satisfies $u \in H^{r}(\mathcal{K})$ with $r \geq 1$ and let $\eta$ be defined as in (4.31), then

$$
\begin{aligned}
\|\eta \eta\| & \leq c h^{s-\frac{1}{2}}|u|_{s, \mathcal{K}} \\
\mid] \eta \| & \leq c h^{s-\frac{1}{2}}|u|_{s, \mathcal{K}}
\end{aligned}
$$

for $0 \leq s \leq \min (p+1, r)$.

Proof. Let us develop each term of both norms using the approximation properties of the elementwise $L^{2}$-projection, then

$$
\|\eta\|_{\mathcal{K}} \leq c h^{s}|u|_{s, \mathcal{K}} \quad \text { and } \quad\|\nabla \eta\|_{\mathcal{K}}=|\eta|_{1, \mathcal{K}} \leq c h^{s-1}|u|_{s, \mathcal{K}} .
$$

Thus

$$
\left\|\tilde{h}^{\frac{1}{2}} \beta \cdot \nabla \eta\right\|_{\mathcal{K}} \leq c\left\|\tilde{h}^{\frac{1}{2}} \nabla \eta\right\|_{\mathcal{K}} \leq c h^{s-\frac{1}{2}}|u|_{s, \mathcal{K}}
$$

Finally applying the trace inequality for non-discrete functions, Lemma 2.2, yields

$$
\left\||\beta|^{\frac{1}{2}}[\eta]_{\beta}\right\|_{\mathcal{F}} \leq c\left\|||[\eta]_{\beta}\right\|_{\mathcal{F}} \leq c\left(\left\|\tilde{h}^{-\frac{1}{2}} \eta\right\|_{\mathcal{K}}+\left\|\tilde{h}^{\frac{1}{2}} \eta\right\|_{1, \mathcal{K}}\right) \leq c h^{s-\frac{1}{2}}|u|_{s, \mathcal{K}} .
$$


In the same manner we develop

$$
\|\{\eta\}\|_{\mathcal{F}} \leq c h^{s-\frac{1}{2}}|u|_{s, \mathcal{K}} .
$$

Combining these bounds leads to the result.

Theorem 4.3 (Convergence). Let us denote $u$ the exact solution of the problem (2.1), and $u_{h}$ the solution of the discrete problem (3.1). Further, assume that $u \in H^{r}(\mathcal{K}) \cap H^{1}(\Omega)$ with $r \geq 1$, and that $\beta \in[\operatorname{Lip}(\Omega)(\Omega)]^{2}$. Then

$$
\left.\left|\| u-u_{h}\right|\left|\leq c h^{s-\frac{1}{2}}\right| u\right|_{s, \mathcal{K}}
$$

for $0 \leq s \leq \min (p+1, r)$.

Proof. Let $\eta, \xi_{h}$ be defined by (4.31). Then use the triangle inequality

$$
\left\|u-u_{h}\right\||\leq\|\eta\||+\left|\left\|\xi_{h}\right\|\right| .
$$

By Proposition 4.3 the first term is bounded by

$$
\left\||\eta \|| \leq c h^{s-\frac{1}{2}}|u|_{s, \mathcal{K}} .\right.
$$

For the second term apply the Inf-Sup condition, the consistency, the continuity and the approximability result, Proposition 4.1, Lemma 3.5, Proposition 4.2 and Proposition 4.3,

$$
\begin{aligned}
\left\|\xi_{h}\right\| \mid & \leq c \sup _{v_{h}^{\prime} \in V_{h}^{p}} \frac{a\left(\xi_{h}, v_{h}^{\prime}\right)+j\left(\xi_{h}, v_{h}^{\prime}\right)}{\left\|v_{h}^{\prime}\right\| \mid}=c \sup _{v_{h}^{\prime} \in V_{h}^{p}} \frac{a\left(\eta, v_{h}^{\prime}\right)+j\left(\eta, v_{h}^{\prime}\right)}{\left\|v_{h}^{\prime}\right\| \mid} \\
& \left.\leq c \sup _{v_{h}^{\prime} \in V_{h}^{p}} \frac{\mid] \eta\left[\left|\left\|v_{h}^{\prime}\right\|\right|\right.}{\left\||| v_{h}^{\prime}\right\| \mid}=\mid\right] \eta[\mid \\
& \leq c h^{s-\frac{1}{2}}|u|_{s, \mathcal{K}} .
\end{aligned}
$$

Combining (4.33) and (4.34) leads to the result.

\section{Analysis of the PROJECtion}

We first investigate in the local projection and then build a global projection in a second step based on the local one.

\subsection{Local projection.}

Lemma 5.1 (Local Projection). Let $\kappa \in \mathcal{K}$ be an arbitrary element. For $v_{1} \in L^{2}(\kappa)$ and $v_{2} \in L^{2}(\partial \kappa)$, there exists a unique local projection $\pi_{h}=\pi_{h}\left(v_{1}, v_{2}\right) \in \mathbb{P}_{p}(\kappa)$ such that

$$
\begin{aligned}
\int_{\kappa}\left(\pi_{h}-v_{1}\right) w_{h}=0 & \forall w_{h} \in \mathbb{P}_{p-1}(\kappa), \\
\int_{\partial \kappa}\left(\pi_{h}-v_{2}\right) z_{h}=0 & \forall z_{h} \in \mathbb{P}_{l}(\partial \kappa),
\end{aligned}
$$

for all $0 \leq l \leq\left\lfloor\frac{p+1}{3}\right\rfloor-1$. In addition if $v \in H^{r}(\kappa), r \geq 1$,

$$
\left|v-\pi_{l}(v, v)\right|_{m, \kappa} \leq c h^{s-m}|v|_{s, \kappa}
$$

for $0 \leq s \leq \min (p+1, r)$ and $0 \leq m \leq r$. Additionally the projection satisfies the following stability properties

$$
\left\|\pi_{h}(0, v)\right\|_{\kappa} \leq c\left\|\tilde{h}^{\frac{1}{2}} v\right\|_{\partial \kappa} \quad \text { and } \quad\left\|\pi_{h}(0, v)\right\|_{\partial \kappa} \leq c\|v\|_{\partial \kappa}
$$

for all $v \in L^{2}(\partial \kappa)$. 
Proof. We first investigate in the formulation of the problem and then prove Lemma 5.1 in three steps: i) Existence and uniqueness (Lemma 5.2), ii) Approximability (Lemma 5.5) and iii) Stability estimates (Lemma 5.6).

First fix the reference element $\widehat{\kappa}$ by its vertices $a_{1}=(-1,1), a_{2}=(-1,-1)$ and $a_{3}=(1,-1)$. Let $\Gamma_{i}, i=1,2,3$, denote the faces of $\widehat{\kappa}$ opposite to $a_{i}$ on $\partial \widehat{\kappa}$. The projection is constructed on the reference element and then transformed to the physical element using the affine transformation.

Observe that $\operatorname{dim}\left(\mathbb{P}_{p}(\widehat{\kappa})\right)=\frac{(p+1)(p+2)}{2}$ and $\operatorname{dim}\left(\mathbb{P}_{l}(\partial \widehat{\kappa})\right)=3(l+1)$. Thus the dimension of the trial space is $\frac{(p+1)(p+2)}{2}$ whereas the dimension of the test space for the two conditions $(5.1)$ and $(5.2)$ is $\frac{p(p+1)}{2}+3(l+1)$. Since $3(l+1) \leq p+1$ observe that $\operatorname{dim}\left(\mathbb{P}_{p}(\widehat{\kappa})\right) \geq \operatorname{dim}\left(\mathbb{P}_{p-1}(\widehat{\kappa})\right)+\operatorname{dim}\left(\mathbb{P}_{l}(\partial \widehat{\kappa})\right)$. This means that the uniqueness can not always be guaranteed by the two conditions (5.1) and (5.2). In the family of functions which satisfies the two conditions (5.1) and (5.2) we pick the function $\pi_{h} \in \mathbb{P}_{p}(\kappa)$ which minimizes the $L^{2}$-error.

Let us introduce the following two bilinear forms

$$
a(v, w)=(v, w)_{\widehat{\kappa}} \quad \text { and } \quad b(v, w)=(v, w)_{\partial \widehat{\kappa}},
$$

for all $v, w \in H^{1}(\widehat{\kappa})$. Then the proposed projection is defined by the following problem:

$$
\begin{array}{rlll}
\min _{\pi_{h} \in \mathbb{P}_{p}(\widehat{\kappa})}\left\|\pi_{h}-v_{1}\right\|_{\widehat{\kappa}} & & \\
\text { such that } a\left(\pi_{h}-v_{1}, w_{h}\right) & = & 0 & \forall w_{h} \in \mathbb{P}_{p-1}(\widehat{\kappa}) \\
b\left(\pi_{h}-v_{2}, z_{h}\right) & =0 & \forall z_{h} \in \mathbb{P}_{l}(\partial \widehat{\kappa})
\end{array}
$$

Introducing the Lagrange multipliers for the side conditions we can consider the following equivalent problem:

$$
\begin{aligned}
\text { find } \quad\left(\pi_{h}, \lambda_{h}, \eta_{h}\right) \in \mathbb{P}_{p}(\widehat{\kappa}) \times \mathbb{P}_{p-1}(\widehat{\kappa}) & \times \mathbb{P}_{l}(\partial \widehat{\kappa}) \quad \text { such that } \\
a\left(\pi_{h}-v_{1}, v_{h}\right)+a\left(\lambda_{h}, v_{h}\right)+b\left(\eta_{h}, v_{h}\right) & =0 \quad \forall v_{h} \in \mathbb{P}_{p}(\widehat{\kappa}) \\
a\left(\pi_{h}-v_{1}, w_{h}\right) & =0 \quad \forall w_{h} \in \mathbb{P}_{p-1}(\widehat{\kappa}) \\
b\left(\pi_{h}-v_{2}, z_{h}\right) & =0 \quad \forall z_{h} \in \mathbb{P}_{l}(\partial \widehat{\kappa}) .
\end{aligned}
$$

Before we prove existence and uniqueness of the projection let us introduce the basis functions for the polynomial spaces. For $\mathbb{P}_{p}(\widehat{\kappa})$ we choose the Dubiner basis [13]. Let $\mathbf{k}=\left(k_{1}, k_{2}\right)$ be such that $0 \leq k_{1}, k_{2}$ and $k_{1}+k_{2} \leq p$, then the set $\left\{\phi_{\mathbf{k}}\right\}$ with

$$
\phi_{\mathbf{k}}(x, y)=P_{k_{1}}^{(0,0)}\left(2 \frac{1+x}{1-y}-1\right)(1-y)^{k_{1}} P_{k_{2}}^{\left(2 k_{1}+1,0\right)}(y)
$$

forms a modal basis of $\mathbb{P}_{p}(\widehat{\kappa}) . P_{n}^{(\alpha, \beta)}(x)$ denotes the orthogonal Jacobi polynomial of degree $n$ associated to the weight function $(1-x)^{\alpha}(1+x)^{\beta}$. Thanks to the orthogonality of Jacobi polynomials, one has for $\mathbf{k} \neq \tilde{\mathbf{k}}$,

$$
a\left(\phi_{\mathbf{k}}, \phi_{\tilde{\mathbf{k}}}\right)=0 .
$$

Then the Dubiner basis satisfies the following properties on the faces:

$$
\begin{aligned}
\phi_{\mathbf{k}}(x, y)_{\Gamma_{1}} & =c P_{k_{1}}^{(0,0)}(x), \\
\phi_{\mathbf{k}}(x, y)_{\Gamma_{\Gamma_{2}}} & =(1-y)^{k_{1}} P_{k_{2}}^{\left(2 k_{1}+1,0\right)}(y), \\
\phi_{\mathbf{k}}(x, y)_{\Gamma_{\Gamma_{3}}} & =(y-1)^{k_{1}} P_{k_{2}}^{\left(2 k_{1}+1,0\right)}(y) .
\end{aligned}
$$


As a basis of $\mathbb{P}_{l}(\partial \widehat{\kappa})$ we choose the set $\left\{\psi_{\mathbf{s}}\right\}$, with $\mathbf{s}=\left(s_{1}, s_{2}\right)$ and $1 \leq s_{1} \leq 3$, $0 \leq s_{2} \leq l$, such that

$$
\begin{aligned}
& \psi_{\left.\mathbf{s}\right|_{\Gamma_{1}}}=P_{s_{2}}^{(0,0)}(x) \text { and } \psi_{\mathbf{s}_{\Gamma_{i}}}=0 \text { for } i=2,3 \text { if } s_{1}=1, \\
& \psi_{\left.\mathbf{s}\right|_{\Gamma_{2}}}=2^{-1 / 2}(1-y)^{s_{2}} \text { and } \psi_{\mathbf{s}_{\Gamma_{i}}}=0 \text { for } i=1,3 \text { if } s_{1}=2, \\
& \psi_{\left.\mathbf{s}\right|_{\Gamma_{3}}}=(1-y)^{s_{2}} \text { and } \psi_{\mathbf{s}_{\Gamma_{i}}}=0 \text { for } i=1,2 \text { if } s_{1}=3 .
\end{aligned}
$$

Lemma 5.2 (Existence and uniqueness). The discrete solution $\left(\pi_{h}, \lambda_{h}, \eta_{h}\right)$ of problem (5.3) exists and is unique.

Proof of Lemma 5.2. Writing

$$
\begin{aligned}
\pi_{h} & =\sum_{i=0}^{p} \sum_{k=0}^{i} \pi_{(k, i-k)} \phi_{(k, i-k)} \in \mathbb{P}_{p}(\widehat{\kappa}), \\
\lambda_{h} & =\sum_{i=0}^{p-1} \sum_{k=0}^{i} \lambda_{(k, i-k)} \phi_{(k, i-k)} \in \mathbb{P}_{p-1}(\widehat{\kappa}), \\
\eta_{h} & =\sum_{s_{1}=1}^{3} \sum_{s_{2}=0}^{l} \eta_{\left(s_{1}, s_{2}\right)} \psi_{\left(s_{1}, s_{2}\right)} \in \mathbb{P}_{l}(\partial \widehat{\kappa}),
\end{aligned}
$$

leads to the following problem:

find the vector $\mathbf{U}=(\pi, \lambda, \eta)$ such that

$$
\mathcal{P} \mathbf{U}=\mathbf{b},
$$

where the vector $\mathbf{U}$ is composed by the coefficients of $\pi_{h}, \lambda_{h}$ and $\eta_{h}$ in the above defined basis. The matrix $\mathrm{P}$ is of the form

$$
\mathcal{P}=\left(\begin{array}{cc}
\mathcal{A} & \mathcal{B}^{\top} \\
\mathcal{B} & 0
\end{array}\right)
$$

The square submatrix $\mathcal{A}$ is generated the bilinear form $a(\cdot, \cdot)$ with trial and test space $\mathbb{P}_{p}(\widehat{\kappa})$ whereas the matrix $\mathcal{B}$ is divided again in two submatrices:

$$
\mathcal{B}=\left(\begin{array}{c}
A \\
B
\end{array}\right)
$$

The matrix $\mathrm{A}$ is generated by the bilinear form $a(\cdot, \cdot)$ with trial space $\mathbb{P}_{p}(\widehat{\kappa})$ and test space $\mathbb{P}_{p-1}(\widehat{\kappa})$. B is generated by the bilinear form $b(\cdot, \cdot)$ with trial space $\mathbb{P}_{p}(\widehat{\kappa})$ and test space $\mathbb{P}_{l}(\partial \widehat{\kappa})$.

To show uniqueness and existence of the projection we have to show that the matrix $\mathcal{P}$ is non singular. Since the bilinear form $a(\cdot, \cdot)$ is symmetric and coercive the matrix $\mathcal{A}$ is symmetric and positive definite. In fact, due to the orthogonality of the basis the matrix $\mathcal{A}$ is even diagonal. Therefore it remains to prove that the matrix $\mathcal{B}$ is of full rank.

Lemma 5.3. The matrix $\mathcal{B}$ is of full rank.

Proof of Lemma 5.3. Let us first focus on the submatrix A. The trial space is $\mathbb{P}_{p}(\widehat{\kappa})$ and the test space is $\mathbb{P}_{p-1}(\widehat{\kappa})$. Thus the dimensions of $\mathrm{A}$ are $\frac{(p+1)(p+2)}{2} \times \frac{p(p+1)}{2}$. 
By the orthogonality of the Dubiner basis, A is of the following form:

$$
A=\left(\begin{array}{ccccccc}
* & 0 & \cdots & 0 & 0 & \cdots & 0 \\
0 & * & \cdots & 0 & 0 & \cdots & 0 \\
\vdots & \vdots & \ddots & \vdots & 0 & \cdots & 0 \\
0 & 0 & \cdots & * & 0 & \cdots & 0
\end{array}\right)
$$

where the $*$ denotes non zero entries. Therefore, using condensation, it remains to analyze the part of B which is generated by the trial space $\mathbb{P}_{p}(\widehat{\kappa}) \backslash \mathbb{P}_{p-1}(\widehat{\kappa})$ and test space $\mathbb{P}_{l}(\partial \widehat{\kappa})$. Let us call this matrix $\mathrm{B}_{123}$. Then

$$
\left(B_{123}\right)_{\hat{s}, k+1}=b\left(\phi_{(k, p-k)}, \psi_{\left(s_{1}, s_{2}\right)}\right), \quad 1 \leq \hat{s} \leq 3(l+1), 0 \leq k \leq p
$$

using the relation $\hat{s}=\left(s_{1}-1\right)(l+1)+s_{2}+1$. First let us analyze the part of $\mathrm{B}_{123}$ corresponding to $1 \leq \hat{s} \leq l+1$, resp. $s_{1}=1,0 \leq s_{2} \leq l$. Note that this part corresponds to the conditions on the face $\Gamma_{1}$. Additionally observe that the restriction of the Dubiner basis to $\Gamma_{1}$, see (5.4), as well as the basis of $\mathbb{P}_{l}(\partial \widehat{\kappa})$, see (5.7), on $\Gamma_{1}$ are Legendre polynomials, i.e.

$$
\left(B_{123}\right)_{\hat{s}, k+1}=\left(B_{123}\right)_{s_{2}+1, k+1}=b\left(\phi_{(k, p-k)}, \psi_{\left(1, s_{2}\right)}\right)=c\left(P_{k}^{(0,0)}, P_{s_{2}}^{(0,0)}\right)_{\Gamma_{1}}=\delta_{s_{2}, k}
$$

for all $0 \leq s_{2} \leq l, 0 \leq k \leq p$ by orthogonality of the Legendre polynomials. Thus the matrix $\mathrm{B}_{123}$ is of the following form

$$
B_{123}=\left(\begin{array}{cc}
D_{1} & 0 \\
* & B_{23}
\end{array}\right) \text {. }
$$

where $\mathrm{D}_{1} \in \mathbb{R}^{(1+1) \times(1+1)}$ is diagonal and $\mathrm{B}_{23} \in \mathbb{R}^{2(1+1) \times(\mathrm{p}-1)}$. Once again we reduce the system by condensation. It remains to prove that the matrix $B_{23}$ is of full rank. First we focus on the upper half of the matrix $B_{23}$, let us call it $B_{2} \in \mathbb{R}^{(1+1) \times(p-l)}$, defined by

$$
\left(B_{2}\right)_{\hat{s}, \hat{k}}=b\left(\phi_{(k, p-k)}, \psi_{\left(2, s_{2}\right)}\right), \quad 0 \leq s_{2} \leq l, l+1 \leq k \leq p,
$$

using the relations $\hat{s}=l+s_{2}+2$ (since now $s_{1}=2$ ) and $\hat{k}=k-l . \mathrm{B}_{2}$ corresponds to the conditions on the face $\Gamma_{2}$. Let us develop an explicit formula for the entries of this matrix

$$
\begin{aligned}
\left(B_{2}\right)_{\hat{s}, \hat{k}} & =b\left(\phi_{(k, p-k)}, \psi_{\left(2, s_{2}\right)}\right)=\left(\phi_{(k, p-k)}, \psi_{\left(2, s_{2}\right)}\right)_{\Gamma_{2}} \\
& =\int_{-1}^{1}(1-y)^{k+s_{2}} P_{p-k}^{(2 k+1,0)}(y) d y=2^{k+s_{2}+1} \frac{\left(k+s_{2}\right) !\left(p-s_{2}\right) !}{\left(k-s_{2}\right) !\left(p+s_{2}+1\right) !}
\end{aligned}
$$

using (5.5), (5.8) and (7.391) $)_{4}$ of [15]. Thus

$$
\left(B_{2}\right)_{\hat{s}, \hat{k}+1}=2 \frac{\left(k+s_{2}+1\right)}{\left(k-s_{2}+1\right)}\left(B_{2}\right)_{\hat{s}, \hat{k}}=2 \frac{(\hat{k}+\hat{s}-1)}{(\hat{k}+2 l-\hat{s}+3)}\left(B_{2}\right)_{\hat{s}, \hat{k}} .
$$

This relation will later be useful. Let us come back to the matrix $B_{23}$ which is composed by

$$
B_{23}=\left(\begin{array}{c}
B_{2} \\
B_{3}
\end{array}\right)
$$

where $\mathrm{B}_{3}$ is defined by $\left(\mathrm{B}_{3}\right)_{\hat{\mathrm{s}}, \hat{\mathrm{k}}}=(-1)^{\hat{\mathrm{k}}+1}\left(\mathrm{~B}_{2}\right)_{\hat{\mathrm{s}}, \hat{\mathrm{k}}}$ for all $1 \leq \hat{s} \leq l+1$ and $1 \leq \hat{k} \leq$ $p-l$. This property follows directly from (5.5) and (5.6). Observe that the rank of 
$B_{23}$ is invariant under a permutation of its columns. By choosing a permuation we have that $B_{23}$ is of full rank if and only if the matrix

$$
\left(\begin{array}{cc}
M_{2 \hat{k}-1} & M_{2 \hat{k}} \\
(-1)^{l+1} M_{2 \hat{k}-1} & (-1)^{l+2} M_{2 \hat{k}}
\end{array}\right)
$$

is of full rank. The matrices $M_{2 \hat{k}-1}$ and $M_{2 \hat{k}}$ are defined by

$$
\left(\mathrm{M}_{\nu}\right)_{\hat{s}, \hat{k}}=\left(B_{2}\right)_{\hat{s}, \nu(\hat{k})}
$$

with $\nu(\hat{k})=2 \hat{k}$ and $\nu(\hat{k})=2 \hat{k}-1$ for all $\hat{k}=1,2, \ldots$ such that $1 \leq \nu(\hat{k}) \leq p-l$. Again the matrix defined by (5.13) is of full rank if and only if the matrix

$$
\left(\begin{array}{cc}
M_{2 \hat{k}-1} & 0 \\
0 & M_{2 \hat{k}}
\end{array}\right)
$$

is of full rank. Now, if $M_{2 \hat{k}-1}$ and $M_{2 \hat{k}}$ are both of full rank the matrix $\mathcal{B}$ is of full rank and the proof is complete. The following lemma allows us to conclude.

Lemma 5.4. The matrices $\mathrm{M}_{\nu}$, defined by (5.14), are of full rank.

Now since the matrix $\mathcal{A}$ is positive definite and $\mathcal{B}$ is of full rank, the matrix $\mathrm{P}$ is nonsingular. Therefore the projection exists and is unique.

Proof of Lemma 5.4. First we develop a formula to pass horizontally from one element to the next one in $\mathrm{M}_{\nu}$. By definition of $\mathrm{M}_{\nu}$ and by relation (5.12) we have

$$
\begin{aligned}
\left(\mathrm{M}_{\nu}\right)_{\hat{s}, \hat{k}+1} & =\left(B_{2}\right)_{\hat{s}, \nu(\hat{k}+1)}=\left(B_{2}\right)_{\hat{s}, \nu(\hat{k})+2}=2 \frac{(\nu(\hat{k})+\hat{s})}{(\nu(\hat{k})+2 l-\hat{s}+4)}\left(B_{2}\right)_{\hat{s}, \nu(\hat{k})+1} \\
& =4 \frac{(\nu(\hat{k})+\hat{s}-1)}{(\nu(\hat{k})+2 l-\hat{s}+3)} \frac{(\nu(\hat{k})+\hat{s})}{(\nu(\hat{k})+2 l-\hat{s}+4)}\left(B_{2}\right)_{\hat{s}, \nu(\hat{k})} .
\end{aligned}
$$

Thus

$$
\left(\mathrm{M}_{\nu}\right)_{\hat{s}, \hat{k}}=\frac{1}{4} \frac{(\nu(\hat{k})+2 l-\hat{s}+3)}{(\nu(\hat{k})+\hat{s}-1)} \frac{(\nu(\hat{k})+2 l-\hat{s}+4)}{(\nu(\hat{k})+\hat{s})}\left(\mathrm{M}_{\nu}\right)_{\hat{s}, \hat{k}+1} .
$$

Observe that $\mathrm{M}_{\nu}$ is not necessarily a square matrix, but we know that the number of columns is equal to or larger than the number or rows.

To show that $\mathrm{M}_{\nu}$ is of full rank it remains to show that $\mathrm{M}_{\nu}$ contains a square matrix of dimension $l+1$ which is non singular. We will prove this by induction. First observe that the principal submatrix of $\mathrm{M}_{\nu}$ of order 1 is positive, i.e. $\left(\mathrm{M}_{\nu}\right)_{1,1}=$ $\frac{2^{l+2}}{p+1}>0$. Then assume that the principal submatrix of order $r$ with $1 \leq r \leq$ $l$ is nonsingular which is equivalent with the fact that all its rows are linearly independent. Thus there exists a unique set of coefficients $\left\{\alpha_{\hat{s}}\right\}_{\hat{s}=1}^{r}$, with at least one $\alpha_{\hat{s}} \neq 0$, such that

$$
\left(\mathrm{M}_{\nu}\right)_{r+1, \hat{k}}=\sum_{\hat{s}=1}^{r} \alpha_{\hat{s}}\left(\mathrm{M}_{\nu}\right)_{\hat{s}, \hat{k}}
$$

for all $1 \leq \hat{k} \leq r$. So (5.16) holds in particular for $\hat{k}=r$ :

$$
\left(\mathrm{M}_{\nu}\right)_{r+1, r}=\sum_{\hat{s}=1}^{r} \alpha_{\hat{s}}\left(\mathrm{M}_{\nu}\right)_{\hat{s}, r} .
$$


Now applying relation (5.15) to both sides of (5.17) yields

$$
\begin{aligned}
& \frac{(\nu(r)+2 l-r+2)}{(\nu(r)+r)} \frac{(\nu(r)+2 l-r+3)}{(\nu(r)+r+1)}\left(\mathrm{M}_{\nu}\right)_{r+1, r+1} \\
& =\sum_{\hat{s}=1}^{r} \alpha_{\hat{s}} \frac{(\nu(r)+2 l-\hat{s}+3)}{(\nu(r)+\hat{s}-1)} \frac{(\nu(r)+2 l-\hat{s}+4)}{(\nu(r)+\hat{s})}\left(\mathrm{M}_{\nu}\right)_{\hat{s}, r+1} .
\end{aligned}
$$

Thus

$$
\left(\mathrm{M}_{\nu}\right)_{r+1, r+1}=\sum_{\hat{s}=1}^{r} \alpha_{\hat{s}} \tilde{\alpha}_{\hat{s}}\left(\mathrm{M}_{\nu}\right)_{\hat{s}, r+1}
$$

with

$$
\tilde{\alpha}_{\hat{s}}=\frac{(\nu(r)+2 l-\hat{s}+3)}{(\nu(r)+2 l-r+2)} \frac{(\nu(r)+2 l-\hat{s}+4)}{(\nu(r)+2 l-r+3)} \frac{(\nu(r)+r)}{(\nu(r)+\hat{s}-1)} \frac{(\nu(r)+r+1)}{(\nu(r)+\hat{s})} .
$$

Using that $\hat{s}<r+1$ yields immediately that $\tilde{\alpha}_{\hat{s}}>1$. But for the principal submatrix of order $r+1$ to be singular all $\tilde{\alpha}_{\hat{s}}$ must be equal to 1 in order to satisfy (5.16) with $\hat{k}=r+1$. Thus the principal submatrix of order $r+1$ is nonsingular. The claim follows by induction.

Lemma 5.5 (Approximability). The projection defined by (5.1) and (5.2) in Lemma 5.1, with $v_{1}=v_{2}=v \in H^{r}(\kappa)$, has optimal approximation properties, i.e.

$$
\left|v-\pi_{h}(v, v)\right|_{m, \kappa} \leq c h^{s-m}|v|_{s, \kappa}
$$

for $0 \leq s \leq \min (p+1, r)$ and $0 \leq m \leq r$.

Proof. Again we show the result on the reference element $\widehat{\kappa}$ and conclude the more general result by the affine transformation. Our goal is to apply the Bramble-Hilbert lemma. For this one need to verify that the projection $\pi_{h}(\cdot, \cdot): H^{r}(\widehat{\kappa}) \rightarrow H^{m}(\widehat{\kappa})$ is linear, complete and continuous.

The linearity is obvious. To show that the projection is complete one needs to prove that $\pi_{h}\left(v_{h}, v_{h}\right)=v_{h}$ for all $v_{h} \in \mathbb{P}_{p}(\widehat{\kappa})$. Indeed this property is equivalent to the fact that the projection exists and is unique, or equivalently that the matrix $\mathcal{P}$ in (5.10) is non singular. Finally the continuity is given by the following argument.

Firstly introduce the following triple norm

$$
\|v, w, z\|^{2}=a(v, v)+a(w, w)+b(z, z)=\|v\|_{\widehat{\kappa}}^{2}+\|w\|_{\widehat{\kappa}}^{2}+\|z\|_{\partial \widehat{\kappa}}^{2}
$$

for all $v, w \in L^{2}(\widehat{\kappa}), z \in L^{2}(\partial \widehat{\kappa})$. The wellposedness of the projection is also equivalent to: there exists $\left(v_{h}, w_{h}, z_{h}\right) \in \mathbb{P}_{p}(\widehat{\kappa}) \times \mathbb{P}_{p-1}(\widehat{\kappa}) \times \mathbb{P}_{l}(\partial \widehat{\kappa})$ and $c>0$ such that

$$
\begin{aligned}
c\left\|\pi_{h}, \lambda_{h}, \eta_{h}\right\| & \leq \frac{a\left(\pi_{h}, v_{h}\right)+a\left(\lambda_{h}, v_{h}\right)+b\left(\eta_{h}, v_{h}\right)+a\left(\pi_{h}, w_{h}\right)+b\left(\pi_{h}, z_{h}\right)}{\left\|v_{h}, w_{h}, z_{h}\right\|} \\
& =\frac{a\left(v_{1}, v_{h}\right)+a\left(v_{1}, w_{h}\right)+b\left(v_{2}, z_{h}\right)}{\left\|v_{h}, w_{h}, z_{h}\right\|} .
\end{aligned}
$$

By continuity of the bilinear forms $a(\cdot, \cdot)$ and $b(\cdot, \cdot)$ it follows that

$$
c|| \pi_{h}, \lambda_{h}, \eta_{h}\||\leq|\| v_{1}, v_{1}, v_{2} \| \mid
$$

Finally we conclude by (5.18) with $v_{1}=v_{2}=v \in H^{1}(\widehat{\kappa})$ and the norm equivalence on the reference element that

$$
\left\|\pi_{h}(v, v)\right\|_{m, \widehat{\kappa}} \leq c\left\|\pi_{h}\right\|_{\widehat{\kappa}} \leq c\left\|\pi_{h}, \lambda_{h}, \eta_{h}\right\| \leq c \mid\|v, v, v\| \leq c\|v\|_{1, \widehat{\kappa}} \leq c\|v\|_{r, \widehat{\kappa}} .
$$


Lemma 5.6 (Stability Estimates). The projection defined by (5.1) and (5.2) in Lemma 5.1, with $v_{1}=0$ and $v_{2}=v \in L^{2}(\partial \kappa)$, satisfies the following stability properties

$$
\left\|\pi_{h}(0, v)\right\|_{\kappa} \leq c\left\|\tilde{h}^{\frac{1}{2}} v\right\|_{\partial \kappa} \quad \text { and } \quad\left\|\pi_{h}(0, v)\right\|_{\partial \kappa} \leq c\|v\|_{\partial \kappa} .
$$

Proof. Once again we show the result on the reference element $\widehat{\kappa}$ and conclude the more general result by the affine transformation. From (5.18) we conclude by the norm equivalence on the reference element that

$$
\left\|\pi_{h}(0, v)\right\|_{\widehat{\kappa}} \leq\left\|\pi_{h}, \lambda_{h}, \eta_{h}\right\| \leq \leq \mid\|0,0, v\|\|=c\| v \|_{\partial \widehat{\kappa}} .
$$

Applying the transformation to the physical element $\kappa$ leads to the first estimate of (5.19) with appropriate scaling in $h$.

For the second estimate we firstly observe that the matrix $\mathcal{P}$ in $(5.10)$ is non singular. Thus we can write $\mathbf{U}=\mathcal{P}^{-1} \mathbf{b}$. Additionally define the mass matrix $\mathrm{M}$ generated by the bilinear form $(\cdot, \cdot)_{\partial \widehat{\kappa}}$ with test and trial space $\mathbb{P}_{p}(\widehat{\kappa})$ and define in a global way

$$
\mathcal{M}=\left(\begin{array}{ccc}
M & 0 & 0 \\
0 & 0 & 0 \\
0 & 0 & 0
\end{array}\right)
$$

Then we may write

$$
\left\|\pi_{h}(0, v)\right\|_{\partial \widehat{\kappa}}^{2}=\mathbf{U}^{\top} \mathcal{M} \mathbf{U}=\mathbf{b}^{\top} \mathcal{P}^{-1} \mathcal{M} \mathcal{P}^{-1} \mathbf{b} \leq \rho\left(\mathcal{P}^{-1} \mathcal{M P} \mathcal{P}^{-1}\right) \mathbf{b}^{\top} \mathbf{b} .
$$

where $\rho$ denotes the spectral radius. Let us analyze $\mathbf{b}^{\top} \mathbf{b}$,

$$
\mathbf{b}^{\top} \mathbf{b}=\sum_{s_{1}=0}^{3} \sum_{s_{2}=0}^{l} b\left(v, \psi_{\mathbf{s}}\right)^{2}=\sum_{s_{1}=0}^{3} \sum_{s_{2}=0}^{l}\left(v, \psi_{\mathbf{s}}\right)_{\Gamma_{s_{1}}}^{2} \leq \sum_{s_{1}=0}^{3} \sum_{s_{2}=0}^{l}\|v\|_{\Gamma_{s_{1}}}^{2}\left\|\psi_{\mathbf{s}}\right\|_{\Gamma_{s_{1}}}^{2},
$$

where $\psi_{\mathbf{s}}$ denotes the basis defined by (5.7) - (5.9). By the definition of the functions $\psi_{\text {s }}$ we may estimate $\left\|\psi_{\left(1, s_{2}\right)}\right\|_{\Gamma_{1}}^{2}=\left\|L_{s_{2}}\right\|_{\Gamma_{1}}^{2} \leq 1$ and compute

$$
\left\|\psi_{\left(2, s_{2}\right)}\right\|_{\Gamma_{2}}^{2}=\frac{2^{2 s_{2}+1}}{2 s_{2}+1}, \quad\left\|\psi_{\left(3, s_{2}\right)}\right\|_{\Gamma_{3}}^{2}=\frac{2^{2 s_{2}+1}}{2 s_{2}+1} .
$$

Therefore

$$
\begin{aligned}
\mathbf{b}^{\top} \mathbf{b} & \leq\|v\|_{\Gamma_{1}}^{2}(l+1)+\|v\|_{\Gamma_{2} \cup \Gamma_{3}}^{2} \sum_{s_{2}=0}^{l} \frac{2^{2 s_{2}+1}}{2 s_{2}+1} \\
& \leq\|v\|_{\Gamma_{1}}^{2}(l+1)+\|v\|_{\Gamma_{2} \cup \Gamma_{3}}^{2} 2^{2 l+1}(l+1) . \leq\|v\|_{\partial \widehat{\kappa}}^{2} 2^{2 l+1}(l+1)
\end{aligned}
$$

and thus

$$
\left\|\pi_{h}(0, v)\right\|_{\partial \widehat{\kappa}}^{2} \leq \rho\left(\mathcal{P}^{-1} \mathcal{M} \mathcal{P}^{-1}\right) 2^{2 l+1}(l+1)\|v\|_{\partial \widehat{\kappa}}^{2} .
$$

Once again we apply the transformation to the physical element $\kappa$.

These three Lemmas (5.2, 5.5 and 5.6) build the proof of Lemma 5.1. 
5.2. Global projection. In this section we proof Proposition 2.1 using its local version Lemma 5.1. We shall define the projection $\Pi_{h}$ of Proposition 2.1 elementwise using its local version $\pi_{h}$. For every element $\kappa \in \mathcal{K}$ define

$$
\left.\Pi_{h}\left(v_{1}, v_{2}\right)\right|_{\kappa}=\pi_{h}^{\kappa}\left(v_{1}, v_{2}\right)
$$

where $\pi_{h}^{\kappa}\left(v_{1}, v_{2}\right)$ denotes the projection defined in Lemma 5.1 on $\kappa$. In addition, using the affine transformation we get

$$
\left|v-\Pi_{h}(v, v)\right|_{m, \mathcal{K}}^{2}=\sum_{\kappa \in \mathcal{K}}\left|v-\pi_{h}^{\kappa}(v, v)\right|_{m, \kappa}^{2} \leq c \sum_{\kappa \in \mathcal{K}} h^{2(s-m)}|v|_{s, \kappa}^{2}=c h^{2(s-m)}|v|_{s, \mathcal{K}}^{2} .
$$

In a similar fashion we develop the global stability estimates (2.8) and (2.9).

\section{Numerical EXAMPLES}

In this section we report some basic numerical result for our method applied to the following transport problem. Let $\Omega \in \mathbb{R}^{2}$ be the square $\Omega=(-1,1)^{2}$. The problem consists of seeking $u: \Omega \rightarrow \mathbb{R}$ such that:

$$
\left\{\begin{aligned}
\beta \cdot \nabla u+\mu u & =f, \\
\left.u\right|_{\partial \Omega^{-}} & =g(y),
\end{aligned}\right.
$$

where $\beta=(1,0)^{\top}$ and $\mu$ are constant coefficients.

6.1. $h$-convergence. In this section we compare the $h$-convergence of the classical upwind-method and the method introduced in this paper with $l=\left\lfloor\frac{p+1}{3}\right\rfloor-1$. Polynomial orders $p \in\{2, \ldots, 5\}$ are considered.

6.1.1. Smooth case. We choose $f(x, y)=0$ and $g(y)=\sin \left(\frac{\pi y}{2}\right)$ in the manner that

$$
u(x, y)=e^{-\mu x} \sin \left(\frac{\pi y}{2}\right) \in C^{\infty}(\Omega) .
$$

Observe that $\mu=0.01$ is choosen sufficiently small such that the transport is dominating the reaction. The exact solution of problem (2.1) satisfies $u \in H^{r}(\mathcal{K}) \cap$ $H^{1}(\Omega)$ for all $r \geq 1$. Thus the theoretical accuracy for the numerical approximation becomes

$$
\left\|\left|u-u_{h} \|\right| \leq c h^{p+\frac{1}{2}}|u|_{p+1, \mathcal{K}}\right.
$$

where $u_{h}$ denotes the numerical approximation defined by (3.1).

Figure 1(a) illustrates the the $L^{2}$-norm of the difference between the exact solution $u$ and the approximation $u_{h}$. We note that for all polynomial orders we have superconvergence of the order $h^{\frac{1}{2}}$ for both methods. Note also that the performance of the new method matches that of the standard upwind method with increasing polynomial order.

6.1.2. Irregular case. We now investigate how the method behaves when approximating irregular solutions. In this case the source term is choosen as

$$
f(x, y)=2 e^{(x+1)}+(x+1)^{2.5}+2.5(x+1)^{1.5},
$$

the boundary condition as $g(y)=1$ and $\mu=1$. This gives an exact solution of the form

$$
u(x, y)=e^{(x+1)}+(x+1)^{2.5} \in H^{3-\varepsilon}(\mathcal{K}) \cap H^{1}(\Omega)
$$

for all $\varepsilon>0$.

In this case the DG-method behaves as $u \in H^{3}(\mathcal{K}) \cap H^{1}(\Omega)$ and a rate of $\mathcal{O}\left(h^{2.5}\right)-$ $\mathcal{O}\left(h^{3}\right)$ can be observed as $h$ tends to zero, for all polynomial orders (compare [17]). 


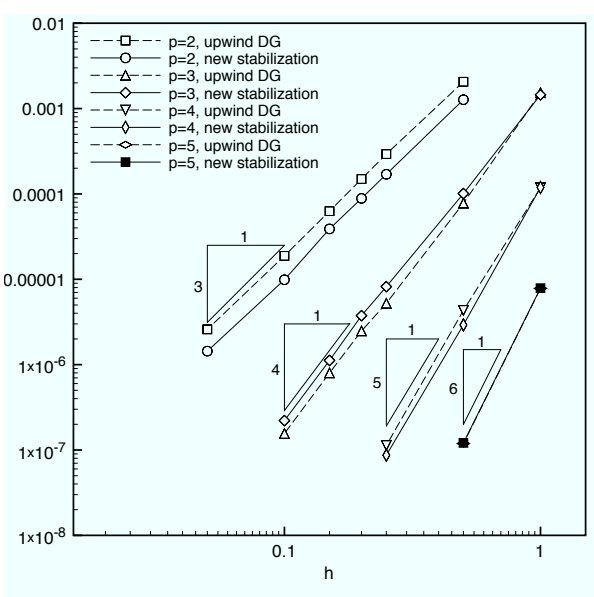

(a) Smooth solution

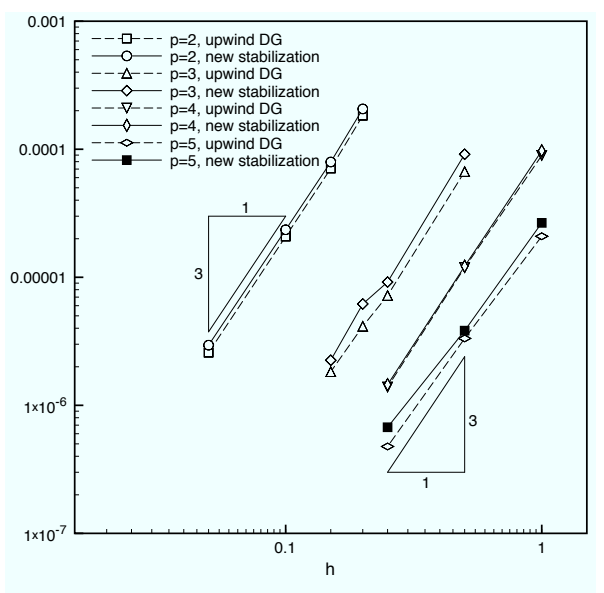

(b) Irregular solution

FiguRE 1. $L^{2}$-norm of error for $h$-refinement and different polynomial orders $p$.

Figure 1(b) illustrates the the $L^{2}$-norm of the difference between the exact solution $u$ and the approximation $u_{h}$. We see that both methods yields similar results.

6.2. Violating the limit for $l$. Here the following pure transport problem is considered. Find $u: \Omega \rightarrow \mathbb{R}$ such that:

$$
\left\{\begin{array}{l}
\beta \cdot \nabla u=0 \\
\left.u\right|_{\partial \Omega^{-}}=g(y)
\end{array}\right.
$$

with

$$
g(y)= \begin{cases}0 & \text { if } \mathrm{y}<0 \\ 1 & \text { otherwise }\end{cases}
$$

The exact solution is then given by $u(x, y)=g(y)$. Observe that no $L^{2}$-coercivity is given and that the theoretical estimates are no longer valid. It is known however that an $L^{2}$-coercivity can be recovered from the convective term using a weighted test function (see [16]).

In this case where the exact solution is discontinuous there is a strong need for stabilization, otherwise spurious oscillations may propagate from the singularity in the whole domain. We consider a fixed $8 \times 8$ unstructured mesh and polynomial order $p=5$. We then compute the solution using four different stabilizations, first without any projection of the jump (similar to upwind stabilization) then using the projection coefficient $l=0, l=1$ and $l=2$. Note that for $l=2$ the stability result no longer holds, since the limit value is given by $\left\lfloor\frac{p+1}{3}\right\rfloor-1=1$ for $p=5$. In Figure 2 we present the four different approximations of the exact solution. Figure 2(a) shows the solution of the method without filtering. Figures 2(b)-(d) illustrates the solutions of the method for the cases $l=0, l=1$ and $l=2$. In all cases spurious local oscillations are present close to the front. However the cases without any projection of the jumps in the stabilization or for $l=0$ or $l=1$, the solutions are qualitatively similar with overshoots of $14 \%, 15 \%$ and $23 \%$ respectively on the 


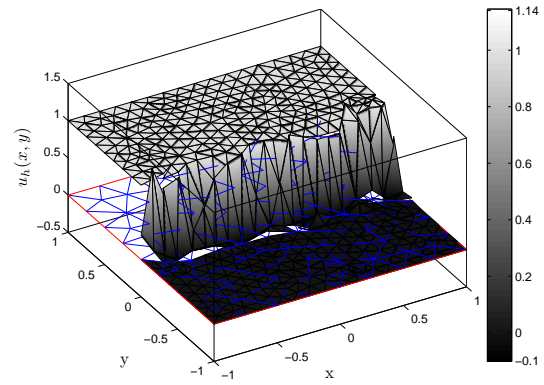

(a) no projection

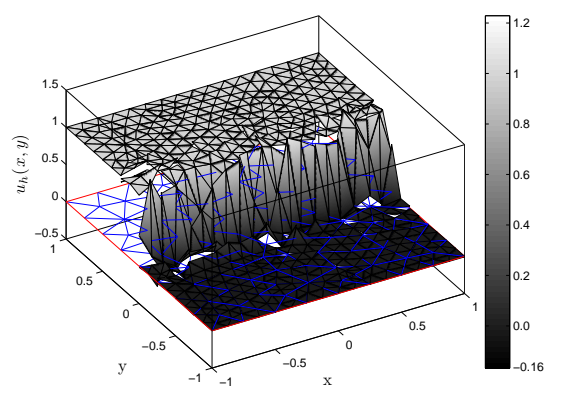

(c) $l=1$

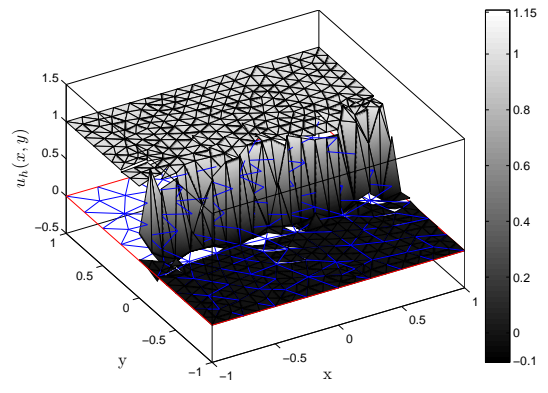

(b) $l=0$

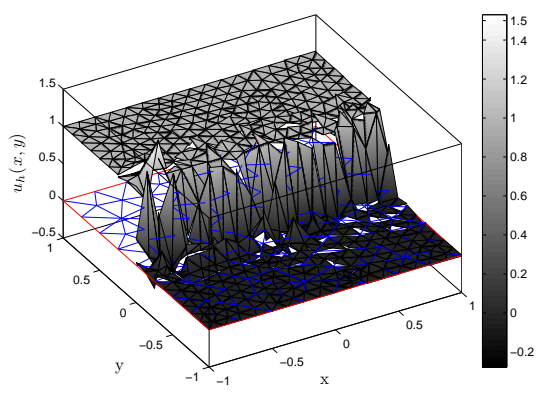

(d) $l=2$

FigURE 2. The approximations of the solution of the problem (6.1) for fixed mesh and polynomial order $p=5$. Figure (a) shows the solution using the method without filtering whereas figures (b)-(d) illustrates the solution using the new approach with $l=0,1,2$.

discontinuity only. In vicinity of the layer the approximate solutions obtained using the projected jumps features larger discontinuities, an effect of the relaxation of the penalization of the low polynomial orders of the jump. This is an interesting feature of our method since the exact solution is indeed discontinuous.

In the case of $l=2$ the theoretical limit for $l$ is violated and in this case the maximum overshoot is $53 \%$ of the exact solution and spurious oscillations are not limited to the elements neighboring to the layer, but strong crosswind propagation of oscillations can be observed (see Figure $2 \mathrm{~d}$ ). This shows that there is a significant loss of stability when the limit value for $l$ is violated indicating that the theoretical limit for $l$ is sharp.

\section{Conclusion}

We have proposed and analysed a discontinuous Galerkin method for the transport equation with local mass conservation properties that are independent of the choice of the stabilization parameter. This is made possible by using a stabilization term that only acts on the projection of the jump of the discrete solution over element faces onto the upper $\frac{2}{3}$ of the polynomial spectrum. Similar a priori error estimates for the convergence in $h$ as for the standard upwind method are 
obtained in spite of the fact that the lowest polynomial modes are unperturbed by the stabilization. This result shows that for high order polynomials on triangles the lowest polynomial modes may be left unstabilized without deterioration of the convergence order for the approximation of smooth solutions. Preliminary numerical tests indicate that the method has similar convergence properties as the upwind method also under refinement in $p$ and that the approximations remain robust for discontinuous exact solutions, even though the local conservation now is independent of the stabilization. Moreover they provide some evidence that the proposed upper bound on $l$ is sharp also in practice.

\section{REFERENCES}

1. F. Brezzi, B. Cockburn, L. D. Marini, and E. Süli, Stabilization mechanisms in discontinuous Galerkin finite element methods, Comput. Methods Appl. Mech. Engrg. 195 (2006), no. 25-28, 3293-3310. MR MR2220920

2. F. Brezzi and M. Fortin, A minimal stabilisation procedure for mixed finite element methods, Numer. Math. 89 (2001), no. 3, 457-491. MR MR1864427 (2002h:65176)

3. F. Brezzi, L. D. Marini, and E. Süli, Discontinuous Galerkin methods for first-order hyperbolic problems, Math. Models Methods Appl. Sci. 14 (2004), no. 12, 1893-1903. MR MR2108234 (2005m:65259)

4. E. Burman, A unified analysis for conforming and nonconforming stabilized finite element methods using interior penalty, SIAM J. Numer. Anal. 43 (2005), no. 5, 2012-2033 (electronic). MR MR2192329

5. E. Burman and A. Ern, Continuous interior penalty hp-finite element methods for advection and advection-diffusion equations, Math. Comp. 76 (2007), 1119-1140.

6. E. Burman and P: Hansbo, Edge stabilization for Galerkin approximations of convectiondiffusion-reaction problems, Comput. Methods Appl. Mech. Engrg. 193 (2004), no. 15-16, 1437-1453. MR MR2068903 (2005d:65186)

7. E. Burman and B. Stamm, Discontinuous and continuous finite element methods with interior penalty for hyperbolic problems., Tech. report, EPFL-IACS report 17, 2005.

8. P. G. Ciarlet, The finite element method for elliptic problems, Classics in Applied Mathematics, vol. 40, Society for Industrial and Applied Mathematics (SIAM), Philadelphia, PA, 2002. MR MR1930132

9. B. Cockburn, Discontinuous Galerkin methods for convection-dominated problems, High-order methods for computational physics, Lect. Notes Comput. Sci. Eng., vol. 9, Springer, Berlin, 1999, pp. 69-224. MR MR1712278 (2000f:76095)

10. _ Devising discontinuous Galerkin methods for non-linear hyperbolic conservation laws, J. Comput. Appl. Math. 128 (2001), no. 1-2, 187-204. MR MR1820874 (2001m:65127)

11. B. Cockburn and C.-W. Shu, Runge-Kutta discontinuous Galerkin methods for convectiondominated problems, J. Sci. Comput. 16 (2001), no. 3, 173-261. MR MR1873283 (2002i:65099)

12. J. Douglas and T. Dupont, Interior penalty procedures for elliptic and parabolic Galerkin methods, Computing methods in applied sciences (Second Internat. Sympos., Versailles, 1975), Springer, Berlin, 1976, pp. 207-216. Lecture Notes in Phys., Vol. 58.

13. M. Dubiner, Spectral methods on triangles and other domains, J. Sci. Comput. 6 (1991), no. 4, 345-390. MR MR1154903 (92k:76061)

14. A. Ern and J.-L. Guermond, Discontinuous galerkin methods for Friedrichs' systems. i. general theory, SIAM J. Numer. Anal. 44 (2006), no. 2, 753-778.

15. I. S. Gradshteyn and I. M. Ryzhik, Table of integrals, series, and products, Academic Press Inc., Boston, MA, 1994. MR MR1243179 (94g:00008)

16. J. Guzmán, Local analysis of discontinuous Galerkin methods applied to singularly perturbed problems, J. Numer. Math. 14 (2006), no. 1, 41-56. MR MR2229818 (2007b:65122)

17. P. Houston, Ch. Schwab, and E. Süli, Discontinuous hp-finite element methods for advectiondiffusion-reaction problems, SIAM J. Numer. Anal. 39 (2002), no. 6, 2133-2163 (electronic). MR MR1897953 (2003d:65108)

18. M. Jensen, Discontinuous galerkin methods for Friedrichs' systems with irregular solutions, Ph.D. thesis, University of Oxford, 2004. 
19. C. Johnson and J. Pitkäranta, An analysis of the discontinuous Galerkin method for a scalar hyperbolic equation, Math. Comp. 46 (1986), no. 173, 1-26. MR MR815828 (88b:65109)

20. P. Lesaint and P.-A. Raviart, On a finite element method for solving the neutron transport equation, Mathematical aspects of finite elements in partial differential equations (Proc. Sympos., Math. Res. Center, Univ. Wisconsin, Madison, Wis., 1974), Math. Res. Center, Univ. of Wisconsin-Madison, Academic Press, New York, 1974, pp. 89-123. Publication No. 33. MR MR0658142 (58 \#31918)

21. W.H. Reed and T.R. Hill, Triangular mesh methods for the neutron transport equation, Tech. Report LA-UR-73-479, Los Alamos Scientific Laboratory, 1973.

22. V. Thomée, Galerkin finite element methods for parabolic problems, Springer Series in Computational Mathematics, vol. 25, Springer-Verlag, Berlin, 1997. MR MR1479170 (98m:65007)

Institute of Analysis and Scientific Computing, Swiss Institute of Technology, LauSANNe, CH-1015, Switzerland

E-mail address: erik.burman@epfl.ch

Institute of Analysis and Scientific Computing, Swiss Institute of Technology, LauSANne, CH-1015, Switzerland

E-mail address: benjamin.stamm@epfl.ch 